\title{
Y-a-t-il un marché de l'eau ?*
}

\author{
Does a water market exist?
}

\author{
par Pascale Babillot \\ IFEN \\ et Philippe Le Lourd, \\ Commissariat Général du Plan
}

Poverty, demographic growth, fast urbanization, depletion of water quality due to the increase of human activities, conduct today to the fact that water is no more a gift from the sky, therefore gratis, but has become a rare ressource for an increasing number of countries. This situation has been recognized at international level as one of the major problems that the XXIst century will have to face necessitating to consider water as an economic good.

What are the values attached to the various functions of water and its uses? Is it possible to consider water as an economic good? Does a water market exist? What are the bases of tarification and pricing of water? Which other concepts are to be taken into account in the future? Those are the questions to which this article try to answer without pretending to be exhaustive. It shows that water is only partially an "economic good" and even less a "commodity" in terms of watervolumes used. It raises the problem of valorization of the non merchant uses of water and new concepts such as "markets of rights" and the "market of virtual water".

\section{INTRODUCTION}

L'eau est un bien vital pour les hommes qui a été longtemps considéré comme une ressource inépuisable et renouvelable, don de la nature, donc gratuit ou dont la valeur a été sous-estimée jusqu'ici. Celle-ci est devenue un bien rare dans un certain nombre de pays, parmi lesquels une majorité de pays en développement. L'accès à l'eau pose pour certains d'entre eux un problème majeur au niveau de leur développement économique futur et de l'allocation de cette ressource entre les différents utilisateurs. La pauvreté, l'accroissement démographique, l'urbanisation rapide, la réduction de la qualité des ressources par l'augmentation des activités humaines devraient accroître cette tendance au $\mathrm{XXI}^{e}$ siècle. L'expérience internationale montre clairement le besoin d'adapter les instruments économiques à la gestion de cette ressource limitée et menacée car cette conception conduit à prendre les valeurs d'usages parmi les critères d'allocation de la ressource. Cela implique aussi, du point de vue de l'environnement, que la gestion de la demande reçoive l'attention voulue et que les coûts imposés aux tiers soient pris en compte ${ }^{1}$. Elle est aussi nécessaire pour des raisons d'équité car ce sont les pauvres qui souffrent le plus du rationnement imposé par une mauvaise gestion des ressources en eau.
Les principes de DUBLIN insistent fortement pour que l'eau soit traitée comme un bien économique et que, en conséquence, les instruments économiques soient de plus en plus largement utilisés, comme les taxes de prélèvement, les redevances de pollution et les marchés de l'eau et que les coûts des usages non marchands soient pris en compte. La récente Conférence internationale sur l'eau et le développement durable qui s'est tenue à Paris, en mars 1998, à l'initiative du Président de la République française, a renforcé cette volonté au niveau politique dans le cadre de la 6ème session de la Commission des Nations-Unies du développement durable qui a choisi en 1998 l'eau comme thème prioritaire.

Quelles sont les valeurs attachées aux différentes fonctions de l'eau et à ses utilisations ? L'eau peut-elle être considérée comme un bien économique? Y-a-t-il un marché de l'eau ? Ya-t-il un marché de la pollution ? Quelles sont les bases de la tarification et du prix de l'eau? Quels autres concepts devraient être pris en compte à l'avenir ?

C'est sur ces différentes questions, aujourd'hui sources de débat, que cet article se propose d'apporter un éclairage sans prétendre faire un examen exhaustif de l'économie de l'eau.

I OCDE (1994).

* Cet article est extrait du livre "L'eau en questions" paru aux éditions Romillat. 


\section{II — L'EAU EST-ELLE UN BIEN ÉCONOMIQUE?}

En tant que bien de consommation (domestique), facteur de production (agricole, industrielle, énergétique...), milieu de vie ou support d'activités in situ (loisirs...), l'eau est un élément indispensable aux activités humaines. En témoigne notamment l'importance des volumes d'eau mis en œuvre par les différents secteurs économiques, en France ou dans le monde.

De ce fait, l'eau acquiert le statut de bien économique pour ce qui concerne la partie où elle est détournée - et/ou utilisée in situ - pour satisfaire les besoins humains, pour être utilisée. Cette condition d'existence de l'eau en tant que bien économique se confirme du fait que sa mobilisation et sa production sont à l'origine de coûts. Ceux-ci, tout en variant fortement en fonction des secteurs d'utilisation ou des pays considérés, peuvent s'avérer non négligeables. Concernant par exemple la production d'eau potable, on estimait déjà en 1987 qu'ils étaient compris entre 0,05 et $4 \$ / \mathrm{m}^{3}$ selon les pays ${ }^{2}$.

Pourtant, l'eau ne peut être assimilée à un bien économique comme les autres, ni même simplement à un bien économique : elle échappe clairement, pour l'économiste, à toute tentative réductrice de sa complexité. En l'occurrence, la première question qui se pose est de savoir si c'est l'eau ("matière première") ou si ce sont les services qu'elle remplit qui doivent en toute rigueur constituer l'objet de l'analyse économique.

L'approche économique "classique" du problème de l'eau, celle qui a régulièrement prévalu dans le passé, et qui domine encore largement aujourd'hui dans les politiques de gestion de l'eau, se rattache à une gestion de l'offre, c'est-à-dire à une démarche de type quantitatif où le problème de l'eau se résout essentiellement à un problème de disponibilités.

Basée sur des évaluations quantitatives des besoins en eau des différents secteurs économiques, cette approche est axée principalement sur les efforts - donc les coûts de la maîtrise de l'eau en tant que ressource (bien renouvelable, mais aussi parfois non renouvelable dans le cas de certains aquifères), et de sa production ou mise à disposition (transport, stockage, traitement... ).

Si cette démarche s'avère utile d'un point de vue financier, elle demeure toutefois trop restrictive car elle suppose implicitement que les usages de l'eau sont équivalents et exclusifs, et que les différents états de l'eau peuvent être réduits à deux termes, les eaux disponibles (c'est-à-dire la ressource) et les eaux usées (ou "non-eau").

Ces aspects sont contredits par les phénomènes liés à l'utilisation de l'eau, et par le rôle général et complexe que celleci joue dans le milieu naturel du fait de sa circulation et de son renouvellement.

Il y a là en effet pour l'analyse économique une difficulté réelle, dont la source réside dans l'opposition existant entre l'unité naturelle que constitue le système hydrologique et la pluralité des usages que l'eau peut remplir dans la sphère des activités économiques, donc la multiplicité des demandes qu'elle permet de satisfaire : un même mètre-cube est susceptible de satisfaire à plusieurs usages successifs au sein d'une même unité de production, cependant qu'il peut faire l'objet de réutilisations différentes entre plusieurs types d'activités.

Une analyse affinée de la demande en eau révèle par conséquent que celle-ci n'est pas relative au bien en tant que tel,

OMS (1987) mais aux différentes et multiples fonctions de l'eau, c'est-àdire aux potentialités que lui confèrent ses propriétés physiques et chimiques et sa répartition dans le milieu naturel. Ces fonctions, lorsqu'elles sont mises en activité, s'expriment dans la pratique par des usages : abreuvement, irrigation, lavage... et ces usages expriment quant à eux les objectifs des utilisateurs : domestiques, agricoles, industriels... ${ }^{3}$

En conséquence, l'objet de l'approche économique ne concerne pas seulement la ressource-eau, mais aussi un actif multi-fonction dont les usages, sollicités par différents secteurs économiques ou extra-économiques, peuvent prendre différentes valeurs et peuvent éventuellement se trouver en concurrence.

Cette compétition est d'autant plus conflictuelle que l'usage, en tant que traduction des différents objectifs de l'utilisateur, ne détruit pas le bien mais peut - et c'est souvent le cas altérer ou détruire une ou plusieurs de ses fonctions, et modifier alors ses possibilités d'utilisations futures. Dans la mesure où l'on a progressivement pris conscience que l'on ne pouvait plus exiger d'un même système naturel qu'est le système hydrographique d'être simultanément offreur (d'une ressource aux potentialités étendues ${ }^{4}$ ) et receveur (d'une eau "usée"), la démarche économique doit tenir compte du fait que la demande en eau sollicite le milieu naturel par une double action : de prélèvement, mais aussi de restitution.

L'importance des retours d'eau usée est loin d'être négligeable dans la mesure où c'est bien l'essentiel de l'eau utilisée qui n'est pas consommé mais restitué au cycle naturel, soit en l'état, soit après une épuration plus ou moins importante. En 1994 en France par exemple, la véritable consommation ("consommation nette") de l'eau ne représentait approximativement que $14 \%$ des volumes prélevés pour toutes les utilisations. Les cœfficients de restitution, bien que variables d'un secteur à l'autre sont assez parlants à cet égard : 10 à $50 \%$ pour l'irrigation, mais 90 à $98 \%$ pour les utilisations industrielles et énergétiques, ou encore 80 à $95 \%$ pour les approvisionnements (urbains) en eau potable 5 .

Tenant compte de cette restitution, l'approche économique des flux utilisés doit par conséquent accorder une valeur particulière aux services rendus par l'eau dans sa fonction d'épuration, valeur dont la perception n'est pas forcément fonction de l'utilisation de l'eau. Les difficultés sont en effet d'autant plus ardues que la demande des qualités de l'eau est en partie disjointe de son utilisation : les pollutions de l'eau, c'est-à-dire ses pertes d'aptitude fonctionnelle, ne sont pas seulement le fait d'agents économiques qui transforment l'eau prélevée en eau usée, elles sont aussi le fait de modes d'occupation de l'espace naturel, ou de certains comportements d'agents non usagers de l'eau mais dont les activités ont des impacts sur l'eau dans son milieu (agriculteurs, industriels, transporteurs, gérants de déchets...).

Il en résulte finalement que l'eau ne peut posséder aucune unicité de valorisation, à cause de la diversité des services qu'elle permet de satisfaire, c'est-à-dire des usages qu'elle rend possibles et qui correspondent à la multiplicité des fonctions employées. Il existe une grande variété de valeurs d'usage, sans rapport avec les coûts de mobilisation et de mise en auvre, ni avec les valeurs marchandes lorsqu'elles existent.

\footnotetext{
A. Erhard-Cassegrain et J. Margat (1982).

‘Y compris la capacité d'auto-épurer - jusqu'à un certain point - les eaux usées retournées.

${ }^{5}$ En milieu rural, le taux est très variable et plus faible : de 0,50 à 0,90 .
} 
Selon la nature, la perception, ou l'intensité de ces valeurs, émergent des exigences différenciées de quantité et de qualité de l'eau, sources potentielles (voire déjà existantes) de conflits en matière d'objectifs.

Cette compétition, qui relève plus de problèmes de conflits de droits d'usage que de problèmes de propriété de l'eau, montre au passage qu'une prise en compte d'un droit d'usage prioritaire sur le droit de propriété devrait aller dans le sens d'une plus grande efficacité économique du point de vue de l'affectation de l'eau vers les services ayant le plus de "valeur".

Le problème, et non l'un des moindres, reste à définir ces valeurs, et ceci indépendamment des principes régissant l'allocation des droits d'usage (répartitions prioritaires, marchés, procédures compensatoires etc.). Cette recherche s'avère inévitable dans la mesure où les approches actuelles de gestion des ressources en eau (approvisionnements à coûts croissants) ne permettront pas de répondre aux besoins des années à venir, notamment dans les grandes agglomérations en expansion, et que les gouvernements sont d'ores et déjà confrontés un peu partout dans le monde aux problèmes complexes (juridiques, culturels etc...) que soulève la réallocation de l'eau entre ses différentes utilisations.

L'idée que les valeurs de l'eau ne sont pas indépendantes des services qu'elle rend (non seulement pour les usages humains mais aussi dans ses rôles naturels) se trouve aujourd'hui à l'origine de multiples recherches économiques visant à attribuer à ces valeurs un contenu monétaire. Ces tentatives de valorisation monétaire à l'égard de composantes hors marché, ou mal prises en compte par le marché, témoignent pour la plupart du souci de rééquilibrer les conditions des choix en matière d'aménagement ou de gestion de la ressource en eau.

Au-delà de l'argumentaire et de la transparence que peut permettre d'apporter l'outil de chiffrage économique, la plasticité intrinsèque de cet instrument pose le problème plus fondamental des systèmes de valeurs (des "politiques") que décide d'adopter la société en matière de préservation des fonctions de l'eau?

\section{III $\square$ Y-A-T-IL UN MARCHÉ DE L'EAU ?}

Si les valeurs d'usage de l'eau sont, bien que tangibles, difficilement monétarisables, les valeurs marchandes de l'eau sont appelées aujourd'hui à jouer le rôle qui leur est traditionnellement dévolu dans une optique économique : révéler la rareté relative de l'eau et jouer un rôle de régulation quantitative et qualitative entre les offres et les demandes en eau.

Ceci nécessite que la confrontation entre offres et demandes puisse s'établir, ce qui n'est le cas que pour une partie minime des eaux actuellement utilisées. Le graphique 1, qui présente le système des offres et des demandes en eau sous la forme d'un système à deux niveaux, situe la part relative des eaux marchandes. Il montre que leur prix ne peuvent régir qu'une partie des échanges entre le milieu et les usagers et ne peuvent jouer que de manière indirecte.

\footnotetext{
${ }^{6}$ G. Meublat (1992).
}

'P. Babillot (1993).

Dans ce système, la rencontre entre l'offre et la demande est :

- soit directe, mais sans marché - donc sans prix - lorsque les usagers exploitants prélèvent l'eau directement dans le milieu naturel. Tel est le cas de la majeure partie des eaux prélevées pour toutes utilisations. En France par exemple, l'ensemble des eaux prélevées par les centrales thermiques (qui s'élevaient à $63 \%$ du total des prélèvements en 1994) ne fait l'objet d'aucune facture pour l'usager, et seule une fraction minime des eaux utilisées par l'industrie et les exploitants agricoles possède un prix (soit respectivement environ $15 \%$ et $1 \%$ des eaux prélevées pour chacun de ces deux postes).

- soit indirecte, lorsque les usagers ne sont pas directement reliés au milieu (ressource), et que s'interpose un secteur intermédiaire de production-distribution d'eau. L'offre en eau (offre 1) du milieu naturel est adaptée aux demandes des usagers (demande 2) par des opérations de prélèvement, d'adduction, de stockage etc. Ces contraintes d'ordre technique sont à l'origine d'une offre interne à la sphère économique (offre 2) de la part des producteurs-distributeurs d'eau.

Du fait de l'existence du secteur intermédiaire de production-distribution, le système ressource/utilisation en eau possède ici une structure à deux niveaux d'offres et de demandes, dont un seul est marchand : un prix s'établit lors de la rencontre de l'offre du distributeur (offre 2) et de la demande de l'usager acheteur (demande 2).

La part de ces eaux marchandes dans le total des eaux prélevées pour toutes les utilisations est faible, de l'ordre de $15 \%$ en France par exemple. Hormis une fraction très faible des eaux destinées à l'irrigation et une part mineure pour l'industrie (indiquées supra), cette eau marchande ne concerne finalement que la totalité de l'eau potable distribuée ${ }^{8}$.

Ces chiffres, qui montrent en résumé qu'il n'existe pas de

sEncore que bien des municipalités ne payent pas l'eau utilisée pour leurs propres services. 
marché de l'eau pour ce qui concerne la majeure partie des quantités d'eau utilisées (les quatre-cinquièmes en France), relativisent par conséquent la portée réelle des mécanismes de marché en matière de gestion de l'eau. Les principes et préceptes avancés à cet égard ("vérité des prix"...) ne concernent que les marchés de distribution d'eau potable ; mais il est vrai cependant que ces marchés drainent à eux seuls des flux financiers non négligeables (on estime par exemple à environ 63 milliards de francs le montant des factures d'eau acquittées par les ménages français en 1995). ${ }^{9}$

Ainsi délimités dans leur composante physique, les "marchés" de l'eau (eau potable) restent très spécifiques dans la mesure où, pour des raisons historiques et techniques, la

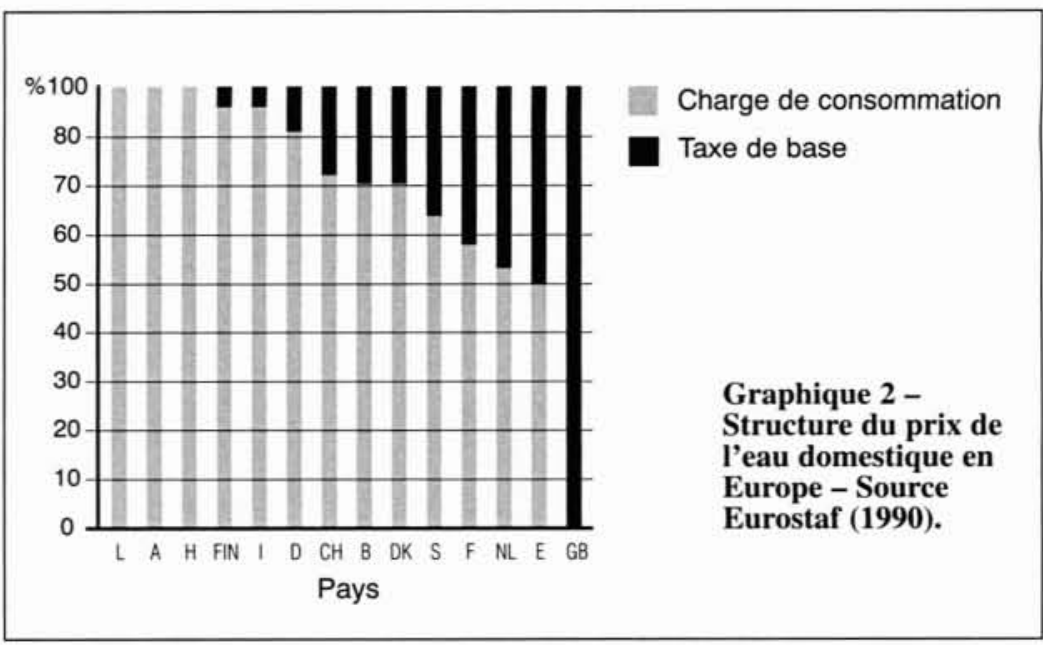
gestion de l'eau est dans presque tous les pays industrialisés de la responsabilité des communes (à l'exception du RoyaumeUni, des Pays-Bas ou de la Finlande), ce qui multiplie le nombre d'instances de décision des prix de l'eau : il existe par exemple plus de 16000 opérateurs de réseaux d'eau potable en France, plus de 6000 en Italie, et en R.F.A. (anciens-länders).

Ce sont donc des pseudo-marchés locaux dans lesquels le consommateur est captif : l'offre en eau, de nature monopolistique, relève avant tout d'un "service public" qui peut être vendu et dont la recette tarifaire reflète alors plus ou moins, selon la politique suivie par l'unité gestionnaire, les coûts de production du service rendu à l'usager desservi. Ainsi estimet-on par exemple que si elle est bénéficiaire en France, l'eau facturée au consommateur ne couvre que $80 \%$ des dépenses en Italie : dans ce pays, une tradition en vigueur jusqu'en 1989 imposait aux prix des services urbains de refléter une politique sociale et non le coût de ces services.

Il convient à cet égard de souligner que le prix de revient du service de distribution d'eau indiqué dans le graphique 1 est un coût direct de production d'eau (pompage, traitement, transport...), qui n'inclut donc pas ou très peu certaines

charges supportées par la puissance publique et répercutées sur les contribuables (investissements initiaux pour les barrages, entretien de la ressource...).

De façon générale, ce coût se répartit entre dépenses indépendantes de la consommation en eau et charges proportionnelles à celle-ci. Cette forme binôme du coût de revient est fréquemment utilisée pour déterminer le prix de vente, dont la double composante pourrait alors être rapprochée de la distinction entre "service public" et "marché" : le terme fixe de la facture d'eau ${ }^{10}$ ("abonnement"...), en assurant la couverture des frais fixes liés à l'existence même du branchement et de l'abonné, représenterait le prix du service de branchement de l'eau, indépendamment de la quantité d'eau utilisée. Il correspondrait en somme plutôt à des dépenses d'investissement. Tandis que l'autre composante de la facture d'eau, terme variable lié au volume consommé, représenterait plutôt le prix du bien et correspondrait davantage aux dépenses de fonctionnement proportionnelles à cette quantité.

Dans cette perspective, charger le terme variable, comme c'est le cas dominant dans la pratique française (suivant des tarifications plates ou dégressives), tendrait à privilégier l'aspect "bien marchand" en entérinant l'idée d'un prix de l'eau,

\section{1. - LE SERVICE DE DISTRIBUTION D'EAU POTABLE EN FRANCE.}

Le service de distribution publique d'eau potable en France est par nature un service public à caractère industriel et commercial et sa gestion est assurée, comme tous les services publics marchands, soit par voie de gestion directe (les régies municipales concernent $25 \%$ des abonnés), soit par voie de gestion déléguée à des sociétés privées $(75 \%)$.

La régie directe consiste à prendre en charge la conception, la construction, le financement et l'exploitation des installations. La délégation peut prendre plusieurs formes : dans le cas des contrats d'affermage (60\% du marché privé), les installations sont financées par la collectivité locale mais leur exploitation ainsi que leur renouvellement sont assurés par le fermier. Celui-ci reverse à la collectivité une surtaxe (incorporée dans le prix de l'eau) pour lui permettre de faire face à ses obligations financières. Les contrats d'affermage sont de durée rarement supérieure à 12 ans. La concession, autre modalité de délégation ( $30 \%$ ), permet à la collectivité de se décharger en totalité de sa responsabilité sur une société privée spécialisée qui réalise, exploite et entretient les installations à ses frais. La durée moyenne d'un contrat de concession est de 20 à 30 ans. Dans les cas de gérance ou de régie intéressée ( $10 \%$ ), la société privée est rémunérée par la commune pour l'exploitation du réseau, avec, dans le deuxième cas, une participation aux résultats et à la fixation des prix. Trois sociétés se partagent en France la gestion déléguée : Vivendi (50\%), Suez Lyonnaise des Eaux (24\%), la SAUR-CISE (groupe Bouygues : $17 \%$ ). 


\section{2. — LA GESTION DE L'EAU EN ANGLETERRE ET AU PAYS DE GALLES'2}

Le système de gestion de l'eau en Angleterre et au Pays de Galles né du Water Act de 1973 devait en théorie promouvoir une totale intégration de manière à tirer parti des interdépendances physiques inhérentes au cycle de l'eau. Dix agences de l'eau, les Regional Water Authorities, dont le territoire correspondait aux bassins hydrographiques importants, furent ainsi créées. Cette organisation permit de concentrer les attributions qui étaient assurées avant 1973 par 1500 opérateurs différents s'occupant, au niveau des municipalités, de tous les aspects relatifs à la gestion de l'eau (entreprises de distribution d'eau, comités d'assainissement, autorités fluviales... ).

A cette intégration des rôles, est venu progressivement s'ajouter le poids croissant d'une centralisation effective, déjà observable au niveau de la loi de 1973 qui avait affaibli le pouvoir des autorités locales. Dix ans plus tard, le Water Act de 1983 devait poursuivre dans cette voie : le Conseil d'Administration (CA) des Waters Authorities, composé de représentants des collectivités locales, des industries et autres usagers et des représentants du gouvernement central, devint entièrement nommé par le gouvernement central. Les autorités locales (élues au suffrage universel) qui avaient auparavant la possibilité d'élire leurs représentants au sein du $C A$, virent ainsi avec cette réforme leur pouvoir se réduire voire disparaitre cependant que dans le même temps le rôle des instances régionales était lui-même faible et ne correspondait pas aux limites de bassin.
Intégrées et centralisées, les Waters Authorities avaient la tâche de tendre vers l'autonomie financière, déchargeant ainsi le secteur public de la responsabilité financière de la gestion de l'eau (après pourtant des décennies de subventions gouvernementales en faveur des gestionnaires locaux). Cette séparation entre la puissance publique et la ressource en eau se radicalise avec la privatisation mise en œuvre par le Water Act de 1989.

Sous la poussée libérale du gouvernement Thatcher réélu en 1983 (qui tendait à réduire l'échelle et les obligations du secteur public), et devant l'effort financier considérable nécessaire pour réhabiliter les réseaux et les centres de traitement de l'eau, le texte de 1989 met en vente les dix autorités régionales, qui assurent $75 \%$ de la distribution d'eau en Angleterre et au Pays de Galles. Celles-ci, devenues des Private Limited Companies, bénéficient d'une structure monopolistique sur leur territoire, chacune restant propriétaire de toutes les installations Elles sont cependant désormais doublement encadrées : la National Rivers Authority voit ses pouvoirs de contrôle réglementaire renforcés et un Office of Water Services est créé afin de suivre les prix de l'eau et les niveaux des services.

Selon certaines estimations, les programmes massifs des investissements (engagés et prévus) devraient conduire les sociétés privées à doubler les taxes relatives à l'eau entre 1990 et 2000 (en livres constantes). bien de consommation. Cette disposition n'est évidemment pas la seule envisageable. Tous les cas peuvent se rencontrer dans le monde, depuis le "tout terme fixe" sans comptage (système britannique avant la privatisation) qui privilégierait à l'inverse l'aspect "service public", jusqu'au "tout terme variable", sans abonnement ni forfait de branchement. Le graphique 2 donne une illustration de la diversité des modes de facturation présente dans les pays d'Europe en 1988 à défaut de données plus récentes.

Le Luxembourg, l'Autriche, et les Pays-Bas facturent le service d'eau uniquement en fonction des volumes consommés, alors que la Grande-Bretagne (avant la privatisation) ne se base que sur une taxe fixe évaluée à partir de la valeur de la propriété foncière. Entre ces deux extrêmes, se situent les autres pays dont la part de la taxe fixe varie entre $4 \%$ (Finlande) ou $5 \%$ (Italie) et $40 \%$ (Espagne) $)^{11}$.

Il reste enfin à observer que si les services de distribution d'eau et d'assainissement restent encore globalement dominés par des formes de gestion relevant d'entités publiques, cette proportion devrait désormais s'inverser au bénéfice de gestionnaires privés, via des formes d'économie mixte ou de gestion déléguée.

Tel est déjà le cas dans certains pays d'Europe, comme en France par exemple, où de grands groupes (Vivendi, Suez Lyonnaise des Eaux...) dominent le marché de l'eau distribuée (75\% de la population desservie) (encadré 1). Plus significative encore est l'expérience anglaise de gestion de l'eau et la récente privatisation des Water Authorities (encadré 2 ).

\footnotetext{
"EUROSTAF (1990)

12 E.C. Penning-Roswel (1990) ; B. Barraqué (1995).
}

La croissance de la participation du secteur privé dans le domaine de l'eau et de l'assainissement est aussi bien observable dans le reste du monde. A la suite de la Côte d'Ivoire, qui apparaît comme un précurseur à cet égard, quelques pays en développement ont fait l'expérience d'une exploitation privée des services de distribution d'eau et d'assainissement (Macao, les principales villes de Guinée... ).

D'autres pays ont adopté une démarche plus progressive, comme au Chili où la compagnie des eaux de Santiago soustraite à des sociétés privées beaucoup d'éléments de son service des eaux (relevé des compteurs, entretien des canalisations, facturation... ). Une participation plus active du secteur privé s'effectue aussi en optant pour des variantes du modèle français, comme par exemple en Amérique Latine, à Buenos Aires ou Caracas (contrats de concession). De façon générale, la tendance à la privatisation s'est s'accélérée durant les années 90 .

Face aux investissements requis par le développement prévisible du secteur eau-assainissement dans le monde (extension des réseaux, construction de nouvelles infrastructures, renouvellement des ouvrages anciens, traitements de l'eau... ), le secteur privé imprime une dynamique propre, qui tout en déchargeant les gestionnaires locaux du souci que représentent ces investissements, peut faire valoir sa technicité et son excellence technologique dans un domaine aux exigences croissantes (urbanisation, contrôle en quantité et qualité, normes plus contraignantes... ).

Cette situation de mixité entre décisionnaires publics et gestionnaires privés témoigne de l'intérêt d'une voie médiane qui n'est pourtant pas exempte de critiques. Le débat, qui se cristallise aujourd'hui sur la facture d'eau, doit être replacé, audelà de l'opportunité souvent contestée des stratégies d'inves- 
tissements lourds, dans le cadre plus global des enjeux actuels de la gestion des services d'eau et d'assainissement.

Dans le cadre d'un "marché international de l'eau", qui est en fait un marché de biens d'équipement et de services (doublé d'un marché financier), beaucoup plus que d'eau elle-même, le secteur intermédiaire de production-distribution d'eau et d'assainissement possède, lorsqu'il est privé, des objectifs propres, similaires à ceux de tout secteur économique marchand, et non pas forcément l'objectif de gérer la ressource "bien commun", sinon pour s'assurer éventuellement de son approvisionnement en matière première.

Les "prix de l'eau" qui apparaissent dans ce marché, en tant que recettes transitant directement des consommateurs-usagers vers les entreprises, donnent à celles-ci une grande autonomie, en partie explicative de leur développement. Si les sommes ainsi collectées visent prioritairement à couvrir les coûts directs supportés par ces sociétés, elles leur permettent aussi, grâce aux bénéfices dégagés par ce secteur d'activité, de mener des stratégies industrielles qui leur sont propres. ${ }^{13}$

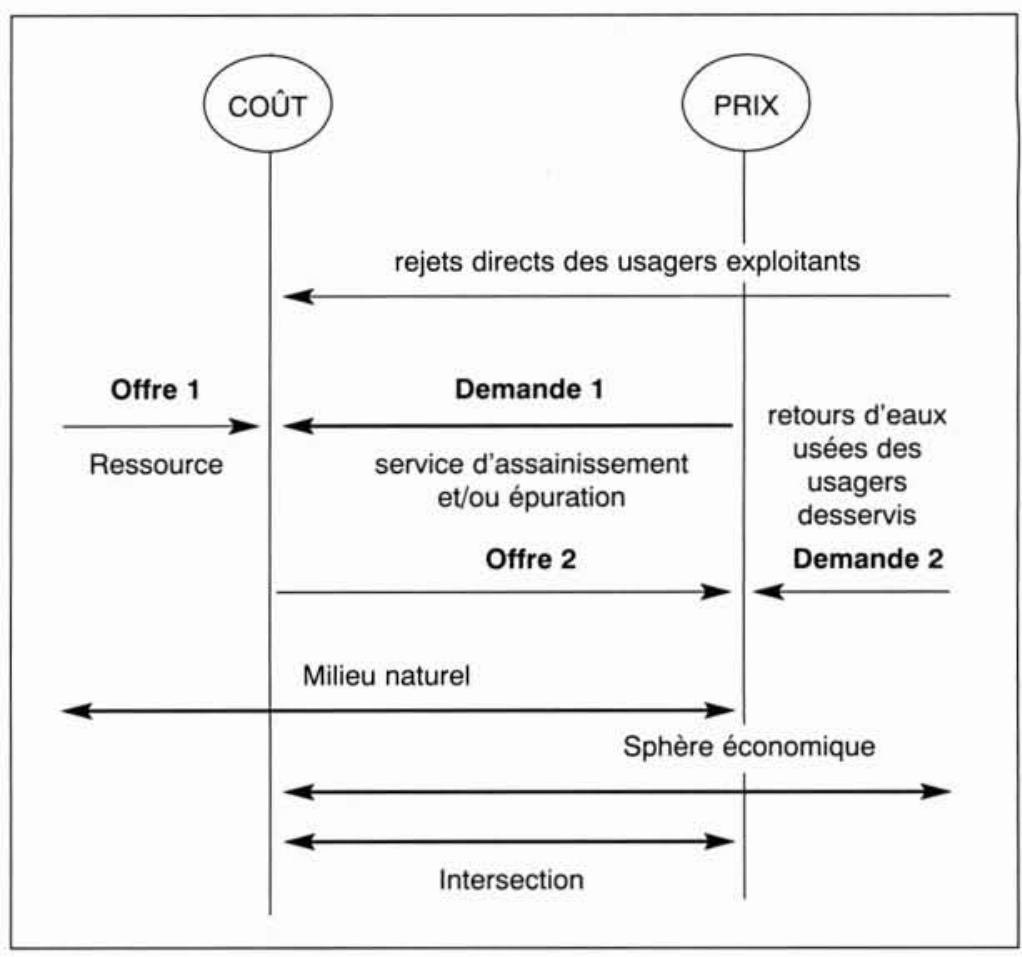

3. Offres et demandes d'assainissement-épuration

\section{3. — LES AGENCES DE L'EAU FRANÇAISES ${ }^{14}$}

La loi de 1964 a créé en France six "Agences Financières de Bassin", dénommées par la suite "Agences de Bassin" et devenues aujourd'hui "Agences de l'Eau". Ces agences, qui ont occupé depuis leur création jusqu'à aujourd'hui une place centrale et originale dans la politique de l'eau française, sont séparées non pas par des limites administratives mais par des limites physiques correspondant en gros aux lignes de partage des eaux entre les différents bassins versants.

Ces organismes sont chargés de participer techniquement et financièrement à la lutte contre la pollution de l'eau et à l'aménagement de la ressource. Leur domaine d'action au titre de la lutte contre la pollution concerne l'aide au financement des stations d'épuration, des réseaux de collecte des eaux usées, ainsi que des centres d'élimination de déchets industriels. Parallèlement, dans le cadre de l'aménagement de la ressource, les agences ont aidé à la construction de barrages-réservoirs et au financement des travaux concernant les unités de production d'eau potable en vue d'améliorer la, sécurité de cette production et la qualité de l'eau distribuée. À partir de 1982, elles ont également aidé au financement des aménagements de rivières. La particularité de ces organismes par rapport aux structures imaginées par les autres pays dans le domaine de l'eau réside en ce qu'ils n'exercent ni le rôle de maîtrise d'ouvrage, ni celui de la réglementation et de la police des eaux. Les agences sont venues s'ajouter aux structures existantes afin d'accélérer ou provoquer les réalisations nécessaires grâce à une incitation technique et financière. Leur vocation de financeur les conduit à monter avec les maitres d'ouvrage des opérations pluri-annuelles dans des conditions favorables, qui ont été renforcées par le Plan National Pour l'Environnement : le sixième programme d'investissement des six agences, approuvé en juin 1992, était de 81 milliards de Francs sur la période 1992-1996, soit un quasi-doublement par rapport à 1987-1991. Le septième programme aidera 105 milliards de Francs de travaux sur la période suivante 1997-2001.

Les agences de l'eau établissent des programmes d'intervention quinquennaux, soumis à la seule contrainte de l'équilibre budgétaire via la perception et l'emploi de redevances de prélèvement et de pollution (ventilées sur la facture d'eau des usagers). Ces redevances sont basées sur des considérations financières (ou "mutualistes") puisqu'elles consistent, à partir du montant global des dépenses prévues dans le programme pluri-annuel, à répartir ce montant entre les différentes catégories de redevables.

Le Conseil d'Administration (CA) élabore le programme d'intervention et en suit l'exécution. II vote chaque année le budget de l'agence en recettes et en dépenses et décide des aides à attribuer aux différents maîtres d'ouvrage (collectivités et usagers privés). Ce programme requiert l'accord du comité de bassin, qui constitue en quelque sorte le parlement du bassin. II est aussi placé sous la tutelle du ministère de l'Environnement et du ministère des Finances.

${ }^{13}$ Pour un aperçu des stratégies d'internalisation et de diversification de la filière française eau-assainissement, voir par exemple Y. N. Genty (1991). 


\section{IV — Y-A-T-IL UN MARCHÉ DE LA POLLUTION ?}

Les aspects qui viennent d'être évoqués quant aux "marchés de l'eau" peuvent être abordés de façon symétrique concernant les retours d'eau après usage qui viennent boucler la filière d'utilisation. Une remarque préalable s'impose cependant : une vision des pollutions de l'eau qui n'est abordée que sous l'angle aval des circuits d'utilisation reste partielle dans la mesure où tous les faits polluants ne sont pas seulement liés aux retours d'eau après usage, de même que tous les pollueurs ne sont pas seulement les usagers de l'eau. Les pollutions engendrées par les pratiques agricoles et par les rejets de déchets notamment, ne sont pas liées aux branches aval des circuits d'utilisation de l'eau et sont sans doute les plus difficiles à contrôler par les mécanismes de marché.

Dans un cadre limité aux offres et aux demandes d'évacuation des eaux usées, il est possible, ici aussi, de faire état d'un double système d'offres et de demandes dans lequel les "prix" ne régissent qu'une partie des flux rejetés.

Lorsque les usagers ne rejettent pas directement les eaux usées dans le milieu naturel (à un coût minimal...), il existe une demande d'évacuation (ou d'assainissement) s'adressant au secteur intermédiaire d'assainissement-épuration ("demande 2"). Ce dernier peut éventuellement ajouter à ce service, dans une proportion très variable, une opération finale d'épuration avant que l'eau ne retourne dans le milieu récepteur et ne bénéficie de ses effets d'auto-épuration naturelle ("demande 1").

Cette double prestation permet de mettre en évidence la nature particulière des différents types de coûts selon qu'il existe ou non un service d'épuration. Dans le cas où il y a assainissement seul, les coûts en question sont des coûts de production directs du service d'évacuation offert aux usagers ; ils peuvent être considérés comme symétriques aux coûts de production d'eau identifiés supra. S'il y a en outre épuration, les coûts résultent alors de l'agrégation des coûts d'évacuation avec ceux de protection et de reconstitution de la ressource en qualité.

Prendre en compte à ce niveau-ci des coûts de "dépollution" doit en principe annuler ("internaliser") une partie des coûts externes générés aussi bien par les usagers de l'eau que par les non-usagers de l'eau (cas des traitements de dénitrification par exemple). Cette prise en compte reste pourtant incomplète puisque, comme on l'a évoqué supra, tous les polluants de l'eau ne transitent pas par ce système mais aussi parce que les incertitudes actuelles (scientifiques notamment) ne permettent pas d'assimiler avec certitude les actions sur les faits polluants (c'est-à-dire les réductions d'émissions, baptisées symptomatiquement "dépollution") aux pollutions résultantes (aux effets in situ sur la qualité des milieux récepteurs).

Lorsqu'ils existent, les "marchés" d'assainissement-épuration, tout aussi locaux et captifs que ceux de l'eau potable, s'établissent lors de la confrontation des utilisateurs-pollueurs avec les unités de gestion de "dépollution". Cette configuration détermine, selon des modalités tout aussi spécifiques que précédemment, des "prix" des services assainissement-épuration. Ceux-ci sont en fait des redevances pour service rendu, ventilées sur la facture d'eau des usagers dans des proportions qui sont extrêmement variables selon les pays (environ $50 \%$ T.T.C. de la facture d'eau française ou $44 \%$ en Italie par exemple).

Parmi ces redevances, il convient de noter la particularité des redevances perçues par les agences de l'eau, qui peuvent apparaître comme des instruments économiques visant à inciter les pollueurs à prendre conscience des coûts externes générés par leurs activités. Dans la pratique cet attachement au principe pollueur-payeur est tempéré par la faiblesse du niveau incitatif des redevances et par le "recyclage" des recettes sous forme de subventions, de prêts ou de primes. Tel est le cas en France où le système mis en place par les agences de l'eau a donné la priorité à une logique de programme ou d'équilibre financier (encadré 3).

Dans les pays en développement, l'insuffisance actuelle des services en matière d'assainissement-épuration rend probable une augmentation des investissements ainsi qu'une croissance de la participation du secteur privé dans ce domaine. On estime par exemple que l'Amérique latine ne traite encore que $2 \%$ des eaux usées, ou que le nombre de citadins qui ne bénéficient pas de l'assainissement a augmenté de plus de 70 millions dans les années 80 , ce qui donne, pour l'ensemble du monde, plus de 1,7 milliard d'individus à n'avoir pas accès à ce type de services ${ }^{15}$.

\section{V — TARIFICATION ET PRIX DE L'EAU}

A la question de savoir jusqu'à quel point les prix constituent un instrument "efficace" de gestion de la ressource en eau, la réponse ne peut être la même selon les objectifs de cette gestion. Cet aspect souligne le caractère relatif de la tarification qui peut répondre à des objectifs différents, en général impossibles à obtenir simultanément et dont la priorité doit par conséquent être clairement précisée.

Parmi ces objectifs, on citera notamment :

- Satisfaire aux obligations financières des vendeurs, agents producteurs-distributeurs d'eau ou service d'assainissement (services publics directs ou délégués à des entreprises privées) qui doivent équilibrer les comptes de leurs services. Les charges doivent ici être couvertes par les recettes, ellesmêmes déterminées par les tarifs .

Dans la plupart des pays industriels, comme dans la majorité des pays européens (Belgique, ex-RFA, Danemark, Royaume-Uni, France, Suisse, Espagne, Pays-Bas), les prix de l'eau sont calculés de façon à couvrir les charges renouvelables afférentes aux services d'approvisionnement en eau et d'assainissement (frais d'exploitation et d'entretien, service de la dette). Les consommateurs paient également la majeure partie des coûts d'investissement dans le cas de l'alimentation en eau, et une part importante et croissante de ces coûts pour l'assainissement (en général plus de la moitié). Certains pays où tel n'est pas le cas (Italie, Luxembourg, Finlande, Norvège... ) font appel pour une part significative à des subventions ${ }^{16}$.

En revanche, dans les pays en développement, les usagers paient beaucoup moins. Une étude de projets financés par la Banque Mondiale a montré que le prix effectif de l'eau ne représente que $35 \%$ environ du coût moyen de la distribution. La part du coût total des projets qui est financée par les entreprises de distribution d'eau confirmerait selon cette même source ces données : leur capacité d'autofinancement ne représente que $8 \%$ du coût des projets en Asie, $9 \%$ en Afrique subsaharienne, $21 \%$ en Amérique Latine et aux Caraibes, et $35 \%$ au Moyen-Orient et en Afrique du Nord ${ }^{17}$.

\footnotetext{
is Banque Mondiale (1992).

16 EUROSTAFF (1990).

${ }^{17}$ Banque Mondiale (1992),
} 
Cependant, dans ces pays, et notamment dans les grandes villes du tiers-monde où se généralise la politique dite des branchements sociaux initiée lors de la "Décennie internationale de l'eau potable et de l'assainissement" (années 80), et soutenue par les organismes internationaux, la logique de rentabilité pose le problème de la charge insurmontable que représente le raccordement pour les populations à revenus faibles et irréguliers.

Une des rares évaluations faite à partir des villes africaines révèle qu'à la faible progression du système moderne de distribution initié pendant la décennie, s'oppose le développement d'un secteur informel de revente d'eau, où les prix peuvent être paradoxalement 8 à 16 fois supérieurs aux tarifs "tranche sociale" pratiqués par les sociétés distributrices pour leurs abonnés aux consommations faibles...

Loin de refléter une "irrationalité" de la population pauvre envers la notion de prix (ou un "consentement à payer" conceptuellement pertinent...), ce type de relation économique met en évidence les conséquences de l'inadéquation de revenus quotidiens et irréguliers à une logique de factures mensuelles ou trimestrielles (à laquelle s'ajoute le coût du branchement qui peut représenter plusieurs mois de revenu médian).

Plus fondamentalement, la généralisation de la vente au micro-détail témoignerait de l'inadaptation de politiques de l'eau - en général fortement intensives en capital - aux conditions de fonctionnement de l'économie urbaine pauvre et à son contexte culturel ${ }^{18}$.

Le problème se pose semble-t-il dans les mêmes termes dans l'ensemble des villes d'Amérique Latine où, compte tenu du mode et du rythme de la croissance urbaine, les services de distribution d'eau et d'assainissement se trouvent dans une situation de déficit chronique. Ainsi le recouvrement des coûts dans le District Fédéral de Mexico a par exemple été estimé à moins de $10 \%$ en 1982 .

Cette situation s'explique en partie par des problèmes tari-

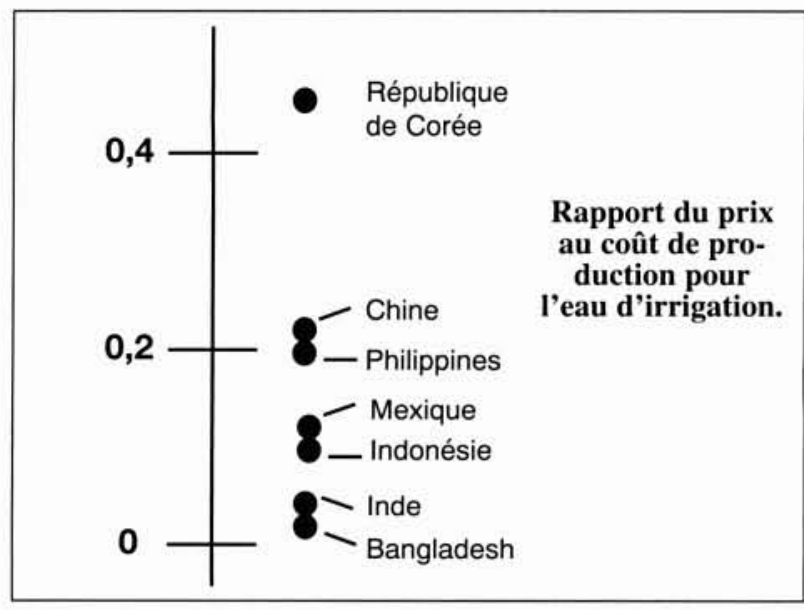

4. Tarif direct de l'eau en proportion de la somme des coûts d'exploitation-entretien et du coût estimatif d'investissement moyen annualisé (diverses années, 1985-88) Source : Banque Mondiale (1992).

faires (les niveaux des tarifs sont jugés trop bas par les organismes prêteurs et des prix parallèles pratiqués par des vendeurs d'eau privés peuvent atteindre 40 fois le prix distribué par le réseau...). Mais elle renvoit aussi à des difficultés plus structurelles qui rendent délicates la maîtrise des coûts et l'amélioration de l'exploitation des services 19.

- La tarification peut aussi permettre d'assurer une fonction de support d'une part variable des coûts imputés à ce niveau par les intervenants publics à responsabilité de gestion des ressources. Ceux-ci ont le pouvoir de répartir une partie des charges communes sur les usagers, via des impositions répercutées (par commodité) sur les tarifs, mais qui peuvent aussi en être disjointes et passer par la fiscalité.

Ces interventions ne concernent pas seulement les usagers desservis et raccordés, mais aussi ceux qui exploitent directe-

is A. Morel à l'Huissier (1991).

${ }^{19}$ D. Faudry (1988)

Tableau 1. - Coût et prix de l'eau dans différents pays du Moyen-Orient (1989, en \$/m3). (Source : N. KHOURI. 1992).

Pays

\section{Chypre (Niçoise,}

Larnacra \&

Limassol SCP)

Égypte (zones urbaines)

Jordanie (Vallée)

Koweit (zones urbaine)

Maroc (Casablanca

\& Doukala)

Arabie Saoudite

(zones urbaines)

Syrie (tout le pays)

Tunisie (tout le pays)

Turquie (Ankara, autres zones urbaines)

\begin{tabular}{|c|c|c|c|c|c|}
\hline \multicolumn{2}{|c|}{ eau portable } & \multicolumn{2}{|c|}{ eau agricole } & \multicolumn{2}{|c|}{ assainissemen } \\
\hline cout' & prix ${ }^{2}$ & cout & prix & cout $^{3}$ & prix \\
\hline 0,71 & 0,58 & 0,69 & 0,15 & (M) 0,27 & 0,11 \\
\hline 0,26 & 0,05 & nd & 0 & nd & 0 \\
\hline 1,15 & 0,77 & 0,23 & 0,01 & (M) 0,37 & 0,08 \\
\hline 2 & 0,50 & nd & nd & (T) 0,4 & 0,03 \\
\hline 0,50 & 0,33 & 0,46 & 0,04 & 0,12 & \\
\hline 2,63 & 0,10 & 0,40 & nd & (T) 0,28 & nd \\
\hline 0,69 & 0,29 & nd & 0 & (S) 0,19 & 0,08 \\
\hline 0,44 & 0,31 & 0,11 & $\begin{array}{c}0,03 \\
\text { à } 0,009\end{array}$ & (S) 0,12 & 0,03 \\
\hline 0,65 & 0,47 & nd & 0,004 & (M) 0,26 & 0,23 \\
\hline
\end{tabular}

Les données de ce tableau proviennent de documents de travail (Banque Mondiale) et de publications et informations fournies par les pays, complétées par celles recueillies en 1997 par la Commission Méditerranéenne du Développement Durable. Aucun essai de standardisation n’a été tenté. Certains chiffres, par exemple, incluent les coûts de transport alors que d'autres ne prennent en compte que les traitements de l'eau distribuée.

Notes

1 - y compris la désalinisation pour le Koweit et l'Arabie Saoudite

2 - valeurs moyennes des structures tarifaires :

3 - (M) : traitement mécanique, généralement secondaire ; $(\mathrm{T})$ traitement tertiaire : (S) bassins de stabilisation

4 - taxes payées pour les services municipaux ou sanitaires. 


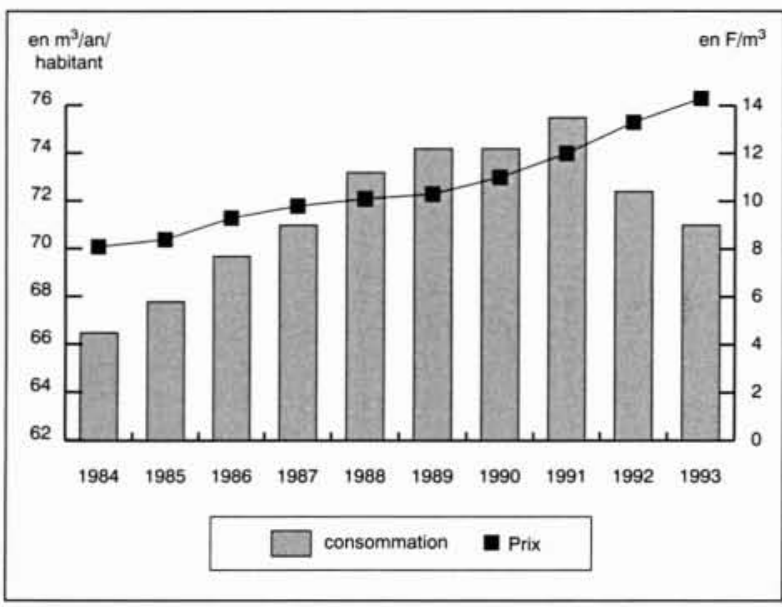

5. Évolution des prix et de la consommation d'eau potable entre 1984 et 1993. Source : CGP à partir de la Direction de l'eau et du rapport de P. Boistard et L. Guérin, op. cit.

ment la ressource et qui sont susceptibles de supporter des taxes et redevances (ou des subventions...) hors de toute tarification de service marchand.

La frontière entre l'usager-consommateur et le contribuable reste très perméable dans la mesure où les modes de financement font l'objet dans la pratique d'un panachage très variable entre taxes, redevances ou subventions de la part des contribuables.

Sous l'effet de l'accroissement des charges et sous l'influence des principes préconisés par de multiples instances internationales (OCDE, Banque Mondiale...) visant à instaurer une "vérité" des prix dans les services publics, l'accélération des travaux d'amélioration des réseaux, de construction de stations d'épuration etc. entraîne dans l'ensemble des pays industrialisés une forte poussée des tarifs en dépit des subventions accordées par les collectivités.

En tout état de cause, dans ces pays, l'usager du service d'eau potable, tout comme celui de l'assainissement, devient le principal financeur de ces services comme le montre le poids de la facture d'eau dans leurs circuits de financement. En 1986 en France, les subventions provenant de l'impôt (État, régions, départements) représentaient 21,2\% des financements des investissements réalisés par les collectivités locales pour l'alimentation en eau et $18,5 \%$ pour l'assainissement. Les moyens financiers pesant sur l'usager (autofinancement, agences de l'eau, FNDAE) représentent respectivement $31,2 \%$ et $23,9 \%$.

Dans le reste du monde, le poids des subventions reste énor$m e$, principalement en ce qui concerne les eaux d'irrigation qui représentent à elles seules $70 \%$ des prélèvements mondiaux en eau. Le graphique 4 montre que dans certains pays fortement demandeurs en eau d'irrigation, les utilisateurs bénéficient d'une subvention qui varie selon les cas de près de $60 \%$ à presque $100 \%$ du coût de production :

Cette distorsion entre coût et prix, attribuable aux subventions, est bien visible pour l'eau d'irrigation. Mais elle l'est aussi pour l'eau potable et même pour l'assainissement comme l'indiquent les données contenues dans le tableau 1 qui s'intéresse à certaines régions du Moyen-Orient et d'Afrique du Nord. La différence révèle que dans tous les cas de figure, le service public subventionné l'emporte de beau${ }^{20}$ Le reste qui est constitué par les emprunts (soit $47,6 \%$ et $57,6 \%$ ), constitue
une participation qui pèse sur l'usager de demain. F. Valiron (1991). coup sur une économie de marché avec "vérité des prix".

- La tarification peut aussi permettre d'assurer une répartition des charges entre les usagers. Outre le fait que le système de tarification peut être utilisé comme un instrument de redistribution entre différentes catégories d'utilisateurs (dans les pays industrialisés, cette fonction de redistribution joue en général en faveur de l'agriculture ou de l'industrie), la répartition des charges peut aussi s'exercer à l'intérieur d'une même catégorie d'usagers. Concernant le service de distribution d'eau potable et d'assainissement, ce dernier aspect suscite régulièrement la question d'une éventuelle péréquation tarifaire entre les usagers de ce service.

Son opportunité est à examiner au regard de l'extrême disparité des prix de l'eau (en France et dans le monde), qui reflète non seulement des différences de situation (état des ressources, conditions d'approvisionnement...) mais aussi des modes très variables de gestion des services (amortissements, prises de bénéfice, programmes de recherche...).

Ainsi un prix moyen, estimé en France à $14 \mathrm{~F} / \mathrm{m}^{3}$ pour l'eau potable en 1995, ne possède qu'une signification très limitée dans la mesure où il masque de fortes disparités locales. Dans le bassin Loire-Bretagne, par exemple, le prix de l'eau varie de $9,2 \mathrm{~F}$ à $23 \mathrm{~F} / \mathrm{m}^{3}$ dans les grandes villes et de $3,3 \mathrm{~F}$ à $30,4 \mathrm{~F}$ dans les autres collectivités en 1995.

De fait, une analyse plus poussée des coûts de revient fait apparaître l'influence des conditions géographiques locales (provenance et qualité de l'eau...), de l'existence ou non de traitements de l'eau (potabilisation, assainissement, épuration...), et aussi de la qualité des différents modes d'exploitation (communale, syndicale, privée...) 21 $^{2}$

De façon générale, l'argument économique se pose fréquemment à l'encontre d'une péréquation menée sur une trop grande échelle. Un tarif unique découragerait les efforts en matière d'économies d'eau tant pour les collectivités à coût de revient élevé - donc subventionnées - que pour celles à coût de revient faible - donc taxées au profit des précédentes.

- La tarification peut aussi avoir comme objectif d'orienter le comportement des consommateurs dans un sens conforme à l'intérêt général. Dans de nombreux pays du monde, la nécessité d'agir sur les demandes en eau semble inéluctable à plus ou moins long terme même si la transformation du cycle de l'eau reste encore principalement conçue sur le principe de développement de l'offre (par intensification à coûts croissants des aménagements et des réseaux de distribution).

Parmi les différentes possibilités d'infléchir les demandes, l'attention est aujourd'hui concentrée sur l'utilisation des instruments économiques, via des modulations du prix de l'eau, conformément au modèle théorique de tarification au côt marginal.

Outre ses difficultés d'application (investissements discontinus, équilibre financier, paramètre unitaire pertinent...), ce principe suppose qu'il existe une bonne corrélation entre le prix de l'eau à l'usager abonné et la consommation de celui-ci. Des études ont pu mettre en évidence la difficulté d'attribuer clairement aux hausses de prix ou aux changements de tarification des modifications des habitudes des usagers dans le passé ${ }^{22}$. En France, le prix de l'eau semble commencer, de manière involontaire, à avoir une fonction incitative ${ }^{23}$ (graphique 5).

21 Boistard (1992 et 1997).

${ }^{22} \mathrm{G}$. Poquet (1996)

${ }^{23}$ Commissariat Général du Plan (1997). 


\section{4. — LE PRIX DU SERVICE DE L'EAU EN FRANCE}

Le prix de l'eau en France prend en compte tout un ensemble d'éléments, qui s'ils sont tous en rapport avec l'eau, dépassent de loin la simple fourniture de ce bien. Sa complexité et la difficulté de son appréhension au niveau national proviennent de la responsabilité donnée aux très nombreuses communes pour les services de distribution d'eau qui créent autant de facturations que de services distincts donc autant de prix de l'eau.

\section{Composition de la facture d'eau}

Toutes les factures d'eau comprennent les éléments suivants : 1) la rémunération du service d'alimentation en eau potable couvrant les coûts de captage, de potabilisation et de distribution mais aussi la gestion des compteurs, les abonnements, les raccordements au réseau...

2) la rémunération du service d'assainissement couvrant la collecte et le traitement des eaux usées.

3) des taxes et des redevances :

- Les redevances de prélèvement $(1 \%)$ et de pollution $(13,5 \%)$ des Agences de l'eau. Ces redevances sont reversées sous forme d'aides aux collectivités locales, aux industriels et aux agriculteurs.

- La redevance du Fonds National pour le Développement des Adductions d'Eau (FNDAE). Ce fonds, géré par le ministère de l'Agriculture, utilise cette redevance pour assurer une péréquation nationale entre les communes urbaines et rurales pour l'alimentation en eau potable et l'assainissement, l'équipement des communes rurales étant beaucoup plus coûteux que celui des zones urbaines.

- La Taxe sur la Valeur Ajoutée (TVA) qui grève tous les produits, y compris l'eau. Depuis 1982 son montant est de 5,5\%. En 1996, la composition de la facture d'eau était la suivante :

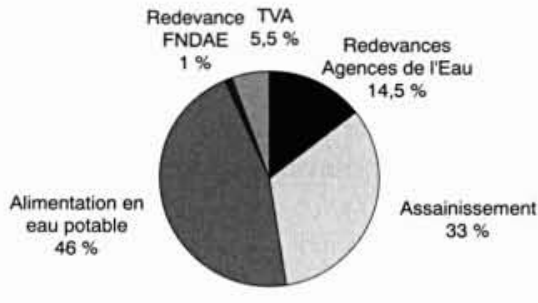

Source : Ministère de l'Environnement

\section{Répartition nationale du prix de l'eau}

En 1995, la distribution des prix de l'eau en France était la suivante :

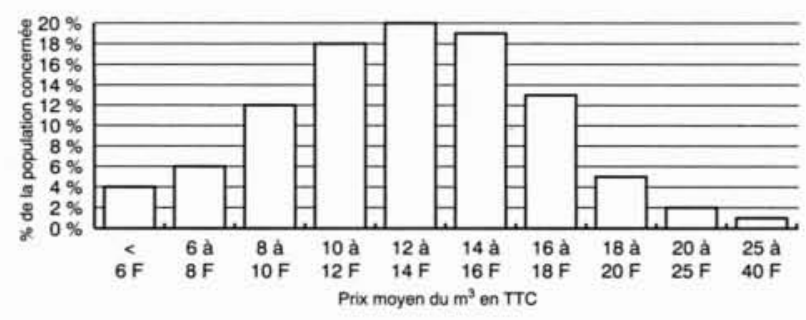

Source : Direction de l'Eau du Ministère de l'Environnement

\section{L'augmentation du prix de l'eau en France}

Le graphique ci-après publié par I'INSEE en 1998, donne au

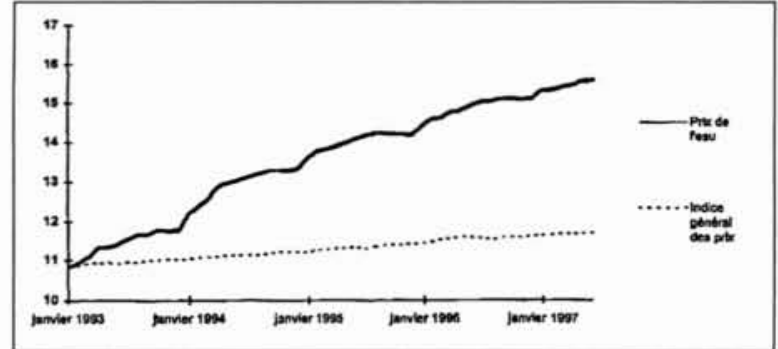

Comparaison du prix de l'eau $\left(\mathrm{F} / \mathrm{m}^{3}\right)$ et de l'indice général des prix depuis janvier 1993.

Prix de l'eau en métropole, sur la base de $120 \mathrm{~m}^{3}$, abonnement inclus, TTC (poste "eau distribuée"). Indice des prix à la consommation y compris tabac, en métropole, pour l'ensemble des ménages. Source : INSEE - Bulletin Mensuel de Statistiques

niveau national une comparaison du prix de l'eau $(\mathrm{F} / \mathrm{m} 3)$ et de l'indice général des prix depuis janvier 1993.

Cette évolution traduit notamment l'effort d'assainissement du pays et l'augmentation des redevances pour être en conformité avec les directives européennes, comme l'indique l'évolution de la composition de la facture d'eau.

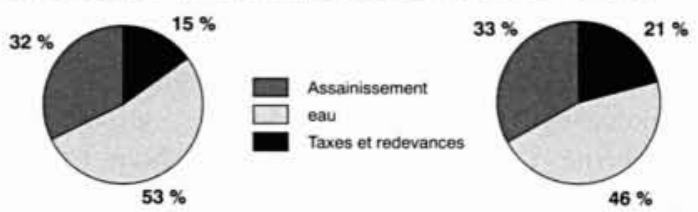

Composition de la facture d'eau en 1991 et en 1996

\section{Observation sur le système français}

1) II n'y a pas de péréquation nationale du prix de l'eau au sens strict en France. L'idée d'un prix unique de l'eau est rejetée par l'ensemble des acteurs. Toutefois la redistribution effectuée par les Agences de l'eau et la FNDAE réalise une péréquation partielle. La différenciation du prix de l'eau, qui traduit les conditions géographiques, existe de fait par les structures éclatées et locales qui gèrent la ressource en eau et de par la règle de l'équilibre budgétaire qui leur est imposée.

2) Les effets du pouvoir de marché sur les prix de l'eau L'autonomie des collectivités locales se heurte directement au pouvoir de marché des opérateurs privés de l'eau, qui influencent assez clairement le prix de l'eau ${ }^{25}$. Le marché de l'eau en France présente en effet une structure oligopolistique avec des tendances monopolistiques marquées sur certaines parties du territoire du fait du nombre limité des sociétés privées existantes.

L'acceptabilité économique de cette situation dépend à la fois de la préservation d'une entrée ouverte pour des groupes concurrents et de l'exercice d'un contrôle public assurant la transparence de l'information et des procédures de passation des contrats délégués.

3) La France cherche aujourd'hui à obtenir une meilleure transparence des factures d'eau et multiplie les observations du prix de l'eau.

Principales sources d'informations: Agences de l'eau, IFEN, INSEE, FNDAE, SPDE, National Utility Service.

${ }^{2}$ Cour des Comptes. Rapport sur la gestion des services publics locaux d'eau et d'assainissement, 1997. pp. 94 à 130. 


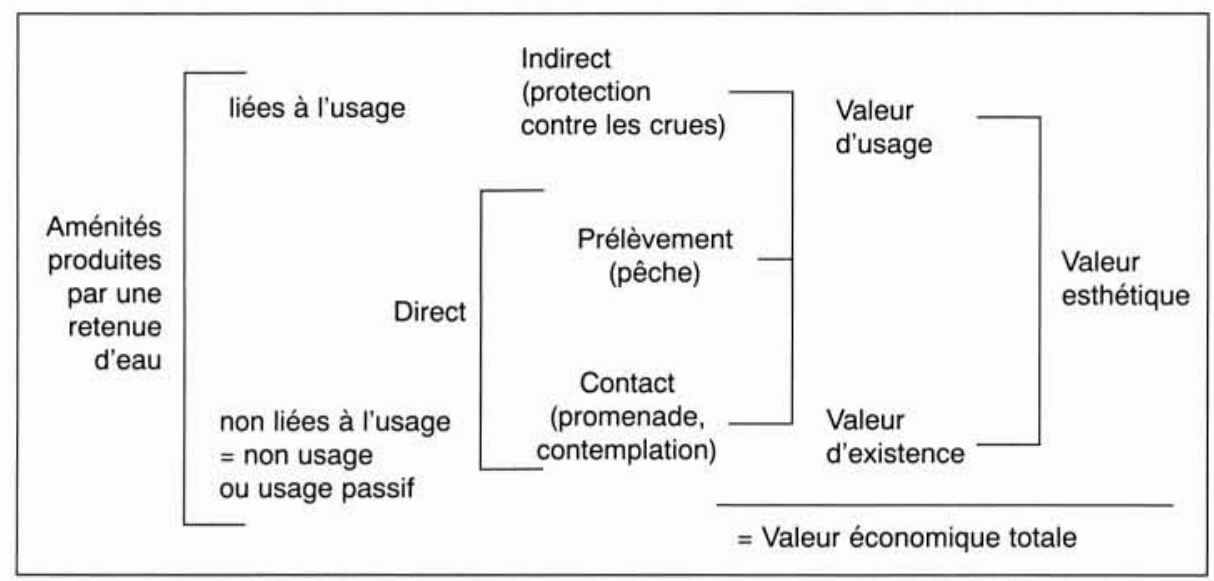

6. Types d'usages et de valeurs liés à la production d'aménités d'une retenue d'eau. Source: Rainelli (1996)
Bien qu'ils restent encore rares, certains calculs d'élasticitéprix menés dans un certain nombre de pays de l'OCDE révèlent une rigidité de la demande aux prix particulièrement nette concernant les usages domestiques de l'eau (les élasticitésprix comprises en général entre $-0,11$ et $-0,40$ ne sont donc pas significativement différentes de zéro) et plus encore pour les seuls usages "intérieurs" de l'eau (élasticité de l'ordre de $-0,04)^{24}$. Concernant les utilisations industrielles de l'eau, le très faible poids actuel des achats d'eau dans les coûts d'exploitation totaux conduit aux mêmes conclusions. Plus significatives apparaissent les élasticités-prix de la demande en eau pour l'irrigation.

\section{VI — QUELS AUTRES CONCEPTS À PRENDRE EN COMPTE À L'AVENIR}

Nous avons vu jusqu'ici les nombreuses difficultés d'une approche économique du bien particulièrement complexe qu'est l'eau. Comment mieux prendre en compte sa qualité de bien social et environnemental à l'avenir ? Sans prétendre être exhaustif, il est intéressant pour finir d'évoquer les avancées récentes de l'idée de marché de l'eau virtuelle, celles sur la valorisation des usages non marchands de l'eau et sur le concept des marchés des droits d'eau et des droits à polluer.

\subsection{Le concept de marché de l'eau virtuelle}

L'eau a été considérée jusqu'ici comme un bien physique matériel. Une nouvelle idée se répand maintenant, celle de l'eau virtuelle pour répondre en particulier à la pénurie de l'eau dans les pays et certaines régions qui en souffrent structurellement. Elle offre en effet une solution à long terme pour des pays comme ceux du Moyen-Orient ou la région méditerranéenne qui ont été les premiers à être touchés par cette situation, qui pose un grave problème d'allocation des ressources en eau. Non seulement ces pays souffrent de la rareté de l'eau mais ce sont ceux qui consacrent l'essentiel de la ressource à la production agricole (jusqu'à $90 \%$, et à 10 fois l' équivalent des eaux domestiques et industrielles réunies) ${ }^{26}$. L'idée est d'utiliser l'eau qui est abondante ailleurs à l'échelle mondiale, non pas à travers un marché global de l'eau, ce qui n'a pas de sens car l'eau est un produit lourd qui ne peut être transporté économiquement sur de longues distances, mais à travers le commerce mondial des produits agricoles eux-mêmes qui font l'économie de quantités considérables d'eau nécessaires à leur production mais employées là où l'eau est disponible. Importer une tonne de blé revient à économiser 1000 tonnes d'eau précieuse, parfois prélevée sur des réserves fossiles ou non renouvelables dans ces pays et à ajouter à leurs ressources l'équivalent en eau virtuelle. De plus le marché mondial du blé est très efficace et subventionné, ce qui est un avantage supplémentaire pour les pays déficitaires en eau et en nourriture. En pratique il va aujourd'hui plus d'eau au Moyen-Orient sous forme virtuelle dans les importations de céréales que celle nécessaire pour la production agricole de l'Égypte.

Cette vision conduit certains analystes, comme $\mathrm{H}$. Shuval, à considérer que le concept de l'auto-suffisance alimentaire pays par pays est dépassé et est non seulement anti-économique mais dangereux car il conduit à une impasse pour l'allocation des ressources en eau génératrice de conflit.

On estime ${ }^{27}$ en effet ce besoin en eau par habitant, production agricole comprise, à $1800 \mathrm{~m}^{3} / \mathrm{an}$, alors que beaucoup de pays du Moyen-Orient disposent globalement de moins de $1000 \mathrm{~m}^{3} / \mathrm{an}$. En revanche, 100 à $150 \mathrm{~m}^{3} / \mathrm{an}$ par habitant sont suffisants pour répondre aux besoins domestiques et industriels et assurer un niveau convenable de confort et de développement économique. Seuls certains pays peu peuplés sont dans une situation de pénurie absolue en dessous de ce seuil (pays du Golfe, Lybie...) et doivent faire appel soit au traitement des eaux usées soit au dessalement de l'eau de mer dont le coût est aujourd'hui acceptable pour les usages domestiques et industriels $\left(1 \$ \mathrm{US} / \mathrm{m}^{3}\right)$. Le marché virtuel de l'eau apparaît donc une voie d'avenir plus économique et soutenable pour une perspective à long terme pour les pays souffrant de pénurie d'eau. Toutefois le fait n'est pas encore largement accepté compte tenu des habitudes acquises jusqu'ici. Il faudra vraisemblablement au moins une génération avant que les pays concernés en tirent tout le bénéfice. Le "marché de l'eau virtuelle" doit s'accompagner en effet d'un marché de biens produits sans eau ou avec très peu d'eau, et exportables par les pays pauvres en eau... Encore faudrait-il alors que les pays exportateurs de biens agro-alimentaires acceptent de faciliter les productions manufacturées (avec peu d'eau) par les pays pauvres en eau et de leur acheter ces produits...

6.2 La valorisation des usages non marchands de l'eau : l'évaluation des actifs naturels

Celle-ci permet d'évaluer des bénéfices économiques et sociaux pouvant être dégagés par la préservation ou l'amélioration de la qualité de l'environnement, en particulier pour les

\footnotetext{
24 OCDE (1987),
}

26 T. Allan (1997).

2) J. Labre (1998) 


\begin{tabular}{|c|c|c|}
\hline - Pour soi-même & - Pour les contemporains & $\begin{array}{l}\text { - Pour les générations } \\
\text { futures (absence d'usage } \\
\text { à l'heure actuelle) }\end{array}$ \\
\hline \multicolumn{3}{|c|}{$\begin{array}{l}\text { Valeur d'option + espérance de surplus = Prix d'option } \\
\begin{array}{c}\text { + valeur de consommation partagée } \\
+ \text { valeur de legs } \\
+ \text { valeur d'existence }\end{array}\end{array}$} \\
\hline & VALEUR DE PRÉSERV & \\
\hline
\end{tabular}

7. Décomposition de la valeur de préservation - l'intérêt d'une conservation d'un actif nature. Source: Rainelli (1996).

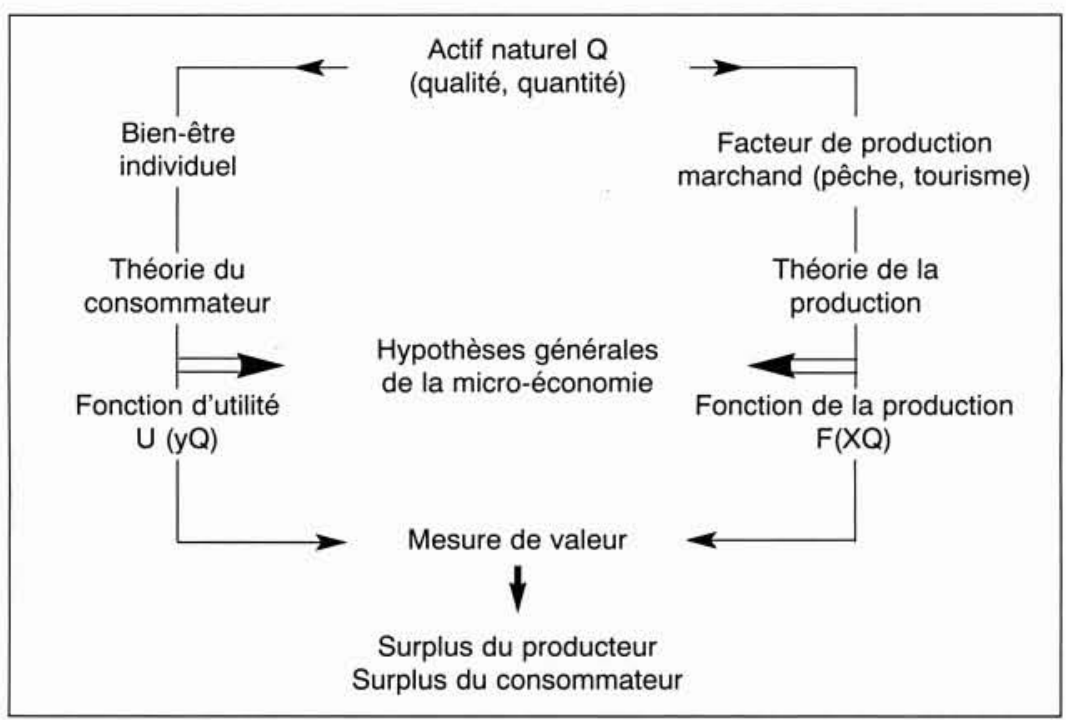

8. Les deux approches complémentaires de la valorisation des actifs naturels Source : Rainelli (1996)

hydrosystèmes. Si ce type d'étude existe depuis déjà une quinzaine d'années dans les grands pays développés (États-Unis, Europe du Nord), la France accuse un certain retard, n'ayant engagé que récemment des travaux dans ce domaine ${ }^{28}$. Une telle évaluation est indispensable à l'heure où les politiques de l'environnement ont pour objectif de promouvoir la conservation et la restauration du milieu aquatique continental et littoral, comme l'affiche par exemple la loi française sur l'eau de 1992. Les coûts élevés d'une telle politique rendent en effet d'autant plus nécessaire d'évaluer précisément les bénéfices qu'elle peut susciter et d'en bien identifier les bénéficiaires.

Dès que l'on aborde la fonction récréative et, plus encore le fonctionnement des écosystèmes, on a un flux de services qui profite à la collectivité. Ce flux de services, qui correspond à la production d'aménités (usages d'agrément rendus par les actifs naturels : contemplation, promenade, qualité de vie) a néanmoins une valeur que l'on peut préciser. A titre d'exemple on trouvera ci-dessus les types d'usages et de valeurs liés à la production d'aménités d'une retenue d'eau (Figure 6).

Cet exemple met en évidence deux grands types d'aménités : celles qui découlent d'un usage et celles qui ne dépendent pas de l'usage du bien.

Les premières proviennent d'un usage direct du milieu aquatique avec un prélèvement comme par exemple dans la pêche ou la chasse au gibier d'eau. La promenade, la contem-

\footnotetext{
28 J-P. Amigues, F. Bonnieux, P. Legoffe, P. Point (1995) et Rainelli (1996).
}

plation d'un site, la baignade ou le canotage relèvent aussi de l'usage direct, comme les activités productives. Le rôle de protection contre les crues joué par une retenue fournit un autre type d'aménités qui correspond à un usage indirect.

Les secondes ne dépendent pas de l'usage direct ou indirect de la ressource en eau mais d'un usage passif fondé sur la valeur intrinsèque du bien, appelée valeur d'existence, liée à l'intérêt porté à la simple connaissance de l'existence de l'actif naturel. La conservation du bien peut être considérée souhaitable pour les générations futures, ce qui détermine la valeur de legs. Enfin, on peut aussi préserver l'actif pour soi (valeur d'option) ou pour ses contemporains (valeur de consommation partagée). L'ensemble constitue la valeur de préservation (Figure 7).

Dans son désir de conserver personnellement un bien environnemental, le consommateur, ignorant comment les choses vont évoluer dans le futur, est prêt à verser une certaine somme (la valeur d'option) qui correspond à une prime d'assurance pour être sûr d'avoir ce bien. Cette valeur d'option s'ajoute au surplus du consommateur qu'il est en droit d'attendre. Le total constitue le prix d'option. Bien qu'il soit difficile de distinguer les composantes du prix d'option et de séparer les différents types d'usage, les schémas 6 et 7 permettent néanmoins de poser les bases d'une réflexion sur les caractéristiques de biens naturels et de la valeur qu'on peut leur attribuer.

$\mathrm{Si}$ on considère dans sa globalité un actif naturel disponible pour une qualité donnée, celui-ci intervient sous deux aspects :

- en tant que facteur de production pour des activités marchandes, comme la pêche ou le tourisme ; on se réfère alors à la théorie de la production en utilisant les concepts traditionnels de fonction de production ;

- en tant qu'élément contribuant au bien-être des individus du fait de l'amélioration qu'apporte à eux-mêmes ou aux générations futures un environnement de qualité ; dans ce cas on a recours à la théorie du consommateur et aux fonctions d'utilité.

\begin{tabular}{|c|c|}
\hline $\begin{array}{l}\text { Approche } \\
\text { Ex-post }\end{array}$ & $\begin{array}{l}\text { Approche } \\
\text { Ex-ante }\end{array}$ \\
\hline basée sur des réalisations & basée sur les intentions \\
\hline$\downarrow$ & $\downarrow$ \\
\hline $\begin{array}{l}\text { - Méthodes des coûts de } \\
\text { déplacement } \\
\text { - Méthodes des prix hédonistes } \\
\text { - Dépenses de protection }\end{array}$ & $\begin{array}{l}\text { - Méthodes d'évaluation } \\
\text { contingente. Interrogation } \\
\text { directe des individus sur } \\
\text { leur préférence }\end{array}$ \\
\hline
\end{tabular}

9. Deux approches pour l'évaluation des biens non marchands. 


\begin{tabular}{|l|l|l|}
\hline & Amélioration & Détérioration \\
\hline $\begin{array}{l}\text { Consentement à payer } \\
\text { Consentement à recevoir }\end{array}$ & $\begin{array}{l}\text { Surplus compensateur } \\
\text { Surplus équivalent }\end{array}$ & $\begin{array}{l}\text { Surplus équivalent } \\
\text { Surplus compensateur }\end{array}$ \\
\hline
\end{tabular}

Tableau 2 . - Définition du consentement à payer et à recevoir.

Ces deux voies aboutissent à une mesure de surplus. Les variations de ce surplus permettent de chiffrer les avantages dus à une amélioration ou à une détérioration de l'actif naturel (Figure 8).

Cette évaluation des biens non marchands liés à l'eau peut être conduite en suivant deux approches (Figure 9) :

- l'approche ex-post est basée sur les coûts de déplacement, le reflet de la qualité de l'environnement sur les variations du prix des biens fonciers (prix hédonistes) et les dépenses de protection engagées par les individus :

- l'approche ex-ante est fondée sur la méthode d'évaluation contingente (MCE) interrogeant directement les individus sur leur préférences et leur consentement à payer (ou à recevoir) pour consommer ou améliorer un actif environnemental au bénéfice de leur bien-être ou vis-à-vis d'une détérioration du milieu (voir Tab. 2)

La méthode MCE fait encore l'objet de débat car il convient de s'assurer que les individus interrogés expriment réellement leurs préférences. Elle suppose aussi qu'il y a bien arbitrage entre la consommation de biens privés et celle de services environnementaux. Elle a l'avantage de valoriser à la fois les bénéfices d'usages et de non usage. Facile d'emploi, elle peut conduire à des évaluations erronées si elle n'est pas parfaitement maîtrisée. Cette approche est en effet délicate du fait de la plasticité intrinsèque de ce type de calcul. Le résultat d'une évaluation contingente tient aux techniques statistiques utilisées et à la manière dont on traite les biais. Par exemple, on élimine systématiquement les réponses de ceux (les déraisonnables...) qui considèrent que la nature a une valeur infinie et qui n'accepteraient aucun équivalent monétaire en échange de sa dégradation, car cette réponse est impossible à prendre en compte dans le calcul.

Ces instruments permettent la prise en compte de la valeur non négligeable des actifs naturels qui sans cela ne sont pas comptabilisés. Leur utilisation dans les choix d'investissements importants comme les grandes infrastructures peut révéler des rentabilités nulles, voire négatives. Ils pourront aider de plus en plus les tribunaux à mieux évaluer les dommages en donnant des valeurs aux biens non marchands.

\begin{tabular}{|c|c|}
\hline Activité récréative & Fourchette probable \\
\hline Pique-nique & $30-120$ \\
Baignade & $70-130$ \\
Bateau à moteur & $50-270$ \\
Bateau sans moteur & $80-350$ \\
Chasse au gibier d'eau & $110-190$ \\
Pêche des salmonidés sédentaires & $100-150$ \\
Pêche des salmonidés migrateurs & $150-350$ \\
Pêche des cyprinidés et percidés & $80-130$ \\
\hline
\end{tabular}

Tableau 3. - Estimation des valeurs récréatives liées à l'eau (en $\mathrm{F} / \mathrm{j})$.
Quelques exemples ${ }^{29}$ peuvent illustrer l'utilité de cette approche :

- Concernant l'alimentation en eau potable, l'approche contingente fournit des évaluations ex-ante particulièrement intéressante du surplus du consommateur. Pour la protection générale de la ressource, les surplus moyens annuels par les ménages se déclarant concernés sont évalués de 250 à $820 \mathrm{~F}$ aux États-Unis et à $496 \mathrm{~F}$ en Allemagne. Si l'on raisonne sur l'ensemble de la population, on est conduit à proposer des valeurs plus basses : de 150 à $480 \mathrm{~F}$ aux États-Unis et de $260 \mathrm{~F}$ en Allemagne. Sur la base de données recueillies, on peut retenir le chiffre de $200 \mathrm{~F}$ par ménage et par an pour la protection générale de la ressource destinée à l'alimentation en eau potable en France. Sur cette base appliquée à la Gironde, on a ainsi vérifié que les bénéfices potentiels sont largement supérieurs au budget de surveillance des nappes (70 MF pour $1 \mathrm{MF}$ ).

- Très récemment, les fonctions d'épuration et de dilution ont été évaluées pour l'ensemble du bassin Adour-Garonne entre 1 et 39 milliards de francs (bénéfice brut annuel). Ces ordres de grandeur montrent la dimension économique significative des services non marchands des hydrosystèmes.

- Autre exemple, l'estimation de la valeur des activités récréatives liées à l'eau. Le tableau 3 donne les valeurs nettes en francs par jour, déduites en France sur la base d'études étrangères existantes.

- Au niveau national, on estime pour la pêche de loisir, en prenant une valeur basse de $100 \mathrm{~F} /$ jour, 12 journées par an et 3 millions de pêcheurs, un surplus total annuel de 3,6 milliards de francs pour ce secteur. Pour l'alimentation en eau potable, la valeur de préservation est estimée à 4,3 milliards de francs/an sur la base de $200 \mathrm{~F} /$ ménage et de 21,5 millions de ménages.

- En décembre 1997, la France a mis en place un groupe de travail "estimations des coûts" pour réfléchir à la gestion des sites NATURA 2000, groupe de travail qui sera conduit à réaliser des valorisations de ce type. Il a en effet à évaluer les coûts pour la collectivité des modes de gestion intensifs (pollutions, perte de biodiversité...) mais aussi les bénéfices collectifs apportés par la gestion écologique de ces espaces (comme les zones humides par exemple).

Les tentatives de valorisation monétaire à l'égard des composantes hors marché témoignent surtout du souci de rééquilibrer les conditions des choix en faveur de l'environnement. ${ }^{30}$

\section{VII $\square$ LE CONCEPT DES MARCHÉS DES DROITS D'EAU ET DES DROITS À POLLUER}

Ce concept a été développé récemment par les économistes de la Banque Mondiale dans le cadre de sa nouvelle politique

${ }^{29} \mathrm{~J}$-P. Amigues, F. Bonnieux, Ph. Le Goffe, valorisation des usages de l'eau et P. Point, séminaire sur la mesure économique des bénéfices attachés aux hydrosystèmes - Paris - mars 1998.

${ }^{\circ}$ G. Meublat (1992) 
pour la gestion des ressources en eau (1993), en réponse aux faiblesses passées des politiques de l'eau, et considérant qu'il fallait passer du rôle traditionnel des gouvernements de fournisseur de tous les services d'approvisionnement en eau à celui de facilitateur et de régulateur pour des services décentralisés, dans le cadre d'une économie libérale. ${ }^{31}$

Il soulève encore de nombreuses interrogations quant à son application dans différents pays du monde, particulièrement en Europe. Quel est son intérêt ? Quelles sont les conditions pour qu'ils soient applicables ? Quels en sont les inconvénients ? Autant de questions légitimes auxquelles nous nous efforcerons de répondre en nous appuyant sur les leçons tirées des marchés existants encore peu nombreux.

\subsection{Quel est l'intérêt des marchés de droit ?}

Ces marchés de droits (aussi appelés marchés de permis négociables) autorisent, pour une quantité globale fixée à l'avance de ressources en eau ou de pollutions, des transactions entre les usagers de l'eau (agriculteurs, municipalités, industriels) qui améliorent, par l'effet de marché, l'allocation des ressources et optimisent les coûts de dépollution. Cet instrument n'implique pas obligatoirement l'État sur le plan financier. Il s'avère plus particulièrement adapté aux situations où les ressources en eau sont rares. Il offre l'avantage dans certains cas de pouvoir transférer des droits non utilisés pour les pollutions ponctuelles pour financer des mesures permettant d'agir par d'autres voies sur les pollutions diffuses.

Il présente différents avantages pour l'environnement et l'économie en théorie ${ }^{32}$ :

\section{- pour l'environnement}

- Les marchés de droits imposent une connaissance précise du niveau global des ressources en eau ou de la pollution sous réserve naturellement des problèmes de contrôle et qu'il n'y ait pas de fraude.

- Les pouvoirs publics ou tout autre agent, tels que les organismes de protection de l'environnement peuvent intervenir en achetant, en vendant ou en thésaurisant des droits.

\section{- pour l'économie}

- Les marchés de droits permettent la réduction du coût économique d'un régime de réglementation et d'autorisation. $\mathrm{Si}$ les permis alloués initialement ne correspondent pas à l'optimum théorique de répartition des efforts, le processus d'échange des droits devrait améliorer l'efficacité économique de l'allocation. L'administration n'a pas besoin de connaître la courbe de coût marginal de réduction des émissions de chaque entreprise, à la différence des systèmes de taxes ou de normes. - Les échanges de droits permettent un développement économique compatible avec la lutte contre la pollution grâce à la possibilité d'achat de permis par les nouvelles activités.

- Les marchés de droits peuvent créer une incitation à limiter la pollution au-delà de la norme administrative, puisque cet effort additionnel peut être rentabilisé par la cession des droits non utilisés.

— Le marché assure un ajustement automatique de l'inflation qui se répercute dans le prix des permis, contrairement au cas des taxes dont le pouvoir incitatif est érodé sans indexation.

- L'industrie est familiarisée avec la logique du marché qu'elle accepte plus facilement que les contraintes fiscales.

\footnotetext{
31 K. William Easter et Robert Hearne (1995).

${ }^{32}$ B. Locatelli, S. Peizerat, K. Sicard et S. Villette (1994).
}

— La possibilité de distribution gratuite des permis aux entreprises évite tout choc économique ainsi que les disparités entre les entreprises qui ont des capacités de financement inégales.

Comme on le voit, ces marchés présentent plus d'intérêt économique qu'environnemental.

\subsection{Quelles sont les conditions pour que les marchés de droits soient applicables ?}

Pour fonctionner, un marché de droits doit répondre à un certain nombre de conditions qui ont été clairement identifiées à partir de l'expérience des marchés existants, essentiellement américains ${ }^{33}$.

- Le bien concerné doit pouvoir faire l'objet d'un marché. Il doit être mesuré, contrôlé et commercialisé. Aussi un marché des droits d'eau peut-il être mis en place seulement si le droit est suffisamment défini quant à la propriété, sa quantité, son caractère mesurable et le degré de confiance qui s'attache à lui. - La demande en eau doit être supérieure à l'offre. Ainsi le marché des droits d'eau est-il un moyen équitable pour que l'eau se reporte vers une utilisation optimale dans le cadre d'une procédure impartiale.

- L'eau fournie à partir d'un droit d'eau doit pouvoir être transportée là où elle est nécessaire et être disponible quand on en a besoin. - Les acheteurs doivent avoir la certitude qu'ils recevront l'eau et seront en mesure d'utiliser leur droit. Cela suppose la mise en œuvre d'un système élaboré de réglementation, d'enregistrement et de surveillance reconnu par le marché.

- Le système doit avoir prévu un mécanisme pour la résolution des conflits.

- Il doit aussi réguler les approvisionnements en fonction des périodes de manques ou d'excès des ressources en eau.

- Il importe que la confiscation ou le transfert du droit d'usages prioritaires soit assorti d'une indemnité compensatrice s'appuyant sur un mécanisme et des critères clairement définis.

- Avant d'instaurer un système de marché des droits, il est essentiel de s'assurer qu'il sera accepté dans le contexte socioculturel de l'endroit où il est envisagé de le mettre en place.

- Le système mis en place doit être viable sur le plan financier.

La réunion de toutes ces conditions limitent quelque peu la mise en place de marchés de droits, comme nous le verrons.

\subsection{Quels sont les inconvénients des marchés de droits ?}

Si les marchés de droits ont des avantages comme nous l'avons vu plus haut, ils ont aussi un certain nombre d'inconvénients.

- Ils présentent une certaine complexité qui semble difficile à éviter totalement et qui vient du besoin de réglementer et de surveiller les transactions. C'est un dilemne car trop de complexité entrave le fonctionnement et un contrôle insuffisant peut conduire à des dérapages.

- La lourdeur et la rigidité du mécanisme à mettre en place se prête mal à ses adaptations ultérieures sans nuire à son efficacité incitative.

- Le niveau optimal d'émission de permis est difficile à déterminer puisqu'il faudrait établir une relation fiable entre la pollution émise et la qualité globale de l'environnement et connaître les courbes de coût marginal global du dommage à l'environnement et de coût social marginal d'épuration.

${ }^{3}$ L. Simpson et K. Ringskog (1997). 
- Il est à craindre une certaine opposition politique à l'idée d'achat et de vente de permis de pollutions de la part des mouvements écologistes.

- Le niveaux des prix et des transactions est faiblement prévisible dans un contexte qui n'est pas parfaitement concurrentiel, ce qui atténue l'aspect incitatif du mécanisme, notamment du point de vue de l'innovation technologique.

- L'allocation initiale des droits et la détermination de l'assiette posent un problème important. Lorsqu'il y a allocation initiale gratuite des permis au prorata des émissions actuelles, celle-ci crée une rente de situation au profit des entreprises existantes, au désavantage des nouvelles entreprises et nouveaux investissements. De plus elle n'apporte aucune ressource budgétaire à l'État.

Jusqu'ici des marchés de droits ont été appliqués particulièrement aux États-Unis (Californie, Caroline du Sud, Colorado, Wisconsin) et dans un petit nombre de pays (Canaries, Chili, Mexique) de façon limitée et avec différents succès. Néanmoins, l'augmentation de la rareté des ressources en eau dans le monde annoncée pour le siècle prochain, ainsi que la globalisation des échanges à laquelle nous assistons, conduisent légitimement à considérer les caractéristiques de cet instrument économique pour améliorer dans certains cas le gestion de l'eau et de l'environnement si les conditions sont réunies. Dans le cas de la pollution de l'eau, on retrouve en Europe les caractéristiques techniques rencontrées dans les marchés américains. Un obstacle cependant est celui de l'organisation institutionnelle en Europe et particulièrement en France avec les agences de l'eau, mais aussi les oppositions politiques existantes des mouvements écologistes. Cela pourrait certes favoriser une gestion locale des marchés au sein d'un même bassin géographique, les agences servant alors d'autorité de contrôle et de réglementation. Cependant, il apparaît difficile de changer le statut des agences, organismes puissants qui perdraient alors une grande partie de leur pouvoir et de leur autorité financière. L'Europe est orientée plutôt vers un système de taxes et de redevances. Il est néanmoins significatif que le Parlement Européen ${ }^{34}$ ait demandé très récemment à la Commission "d'étudier l'éventualité de la création d'un marché de l'eau ainsi que les conditions de sauvegarde environnementale dans le cadre desquels le marché pourrait opérer".

\section{VIII — EN CONCLUSION}

L'eau n'est pas un "bien économique" simple, ni simplement un bien économique. La reconnaissance de l'eau comme "bien économique" dans le contexte de l'économie de marché dominant ne doit pas occulter ses particularités sous peine d'un vision réductrice : l'eau reste avant tout un bien d'environnement dont la circulation dans le milieu naturel ainsi que le rôle général et complexe qu'il joue dans la biosphère laissent entier le problème de sa valeur, nécessitant encore des travaux de recherche ; son appropriation et sa jouissance ne sont pas seulement individuelles mais aussi collectives ; son utilisation est limitée et temporaire du fait de sa circulation dans la sphère économique (on consomme les qualités de l'eau et on la retourne au milieu après usage).

En tant que bien économique (bien de consommation, facteur de production ou support d'activité in situ), l'eau ne possède aucune unicité de valorisation, à cause de la diversité des usages qu'elle rend possibles (correspondant à la multiplicité des fonctions employées). Ces valeurs sont sans rapport avec les coûts de mobilisation et d'exploitation, ni avec les valeurs marchandes (les prix) de l'eau.

Si l'eau n'est que partiellement un "bien économique", elle est encore moins un "bien marchand" en termes de volumes utilisés - même si les flux monétaires mobilisés à ce niveau peuvent être relativement conséquents. Il n'existe pas de marché de l'eau pour ce qui concerne la plus grande partie des quantités d'eau utilisées (les 4/5 en France). Celle-ci est prélevée et rendue directement en "libre service" par les usagers dans les rivières ou les nappes souterraines. La mise en perspective "physique" des flux marchands resitue donc la place relative des "marchés" de l'eau, qui n'existent que dans la mesure où un service est rendu : service de production-distribution d'eau et/ou service d'assainissement.

Lorsque l'eau est marchande, il n'existe pas de prix unique : 1 litre d'eau en bouteille est aujourd'hui vendu au même prix que 1000 litres d'eau du robinet ou 10000 litres d'eau d'un canal d'irrigation...

Les tarifications ne consistent pas seulement à établir des prix en fonction des coûts (coûts d'exploitation mais aussi coûts externes), elles peuvent aussi viser à répartir les charges entre les usagers ou encore à influencer les comportements des consommateurs.

La spécificité des "marchés" subordonne le maniement de l'effet-prix à leurs particularismes. Les mécanismes de marchés (et notamment la "vérité des prix", souvent présentée comme la panacée en la matière) ne peuvent contribuer que dans une mesure limitée à réguler la ressource en eau. Leur efficacité et leurs limites posent en termes plus généraux le problème du niveau pertinent de l'imputation équitable des coûts de la politique de "gestion des ressources en eau". Comme l'a souligné la FAO ${ }^{35}$ : "Les critères d'efficacité ou les paramètres économiques ne répondent pas nécessairement aux critères nationaux de bien-être social" en précisant que "les échecs du marché, en ce qui concerne les ressources en eau, sont à mettre en relation avec les coûts externes, les biens publics et les monopoles naturels".

D'autres concepts pourront être mieux pris en compte néanmoins à l'avenir pour améliorer la gestion économique des ressources en eau dans une perspective de développement durable. La notion de marché de l'eau virtuelle, le concept des marchés de droits là où il est applicable, la valorisation des usages non marchands de l'eau, jusque là négligée, qui exprime, en terme monétaire, le poids de l'eau comme bien social et environnemental.

Toutefois si l'eau a un coût et doit entrer plus largement dans la sphère économique comme le rappelait le Président de la République Française lors de la Conférence internationale de Paris en mars 1998, il est nécessaire de prendre en compte des facteurs pas seulement économiques pour assurer une gestion durable des ressources en eau dans le prochain siècle ; c'est ce que propose le projet de programme d'actions prioritaires qui souligne l'importance d'améliorer la connaissance des ressources en eau et des usages, de faciliter le développement des capacités institutionnelles et humaines, de définir des stratégies de gestion durable et d'identifier des moyens de financement appropriés faisant plus appel aux moyens du secteur privé qu'à ceux de plus en plus limités des États. 


\section{BIBLIOGRAPHIE}

[1] ABOUYOUB Hassan - "L'eau et la globalisation des échanges", Premier Forum Mondial de l'Eau, Marrakech, 21-22 mars 1997, 9 p.

[2] ALLAN Tony - "Virtual water: a long term solution for water short Middle Eastern economies", Water and Development Session - British Association Festival of Science, september 1997, 11 p.

[3] AMIGUES Jean-Pierre - BONNIEUX François, LE GOFFE Philippe et POINT Patrick, "Valorisation des usages de l'eau", INRA-ECONOMICA, septembre $1995,112 \mathrm{p}$.

[4] BABILLOT Pascale - "Optimisation économique et lutte contre la pollution de l'eau", Thèse de doctorat ès Sciences Économiques, Université Panthéon-Sorbonne (Paris 1), R. PASSET dir, janv 1993, 325 p.

[5] BANQUE MONDIALE - "Rapport sur le développement dans le monde. 1992, Le développement et l'environnement", Washington, 1992.

[6] BARRAQUE Bernard - "Les Politiques de l'eau en Europe", la Découverte, 1995, $301 \mathrm{p}$.

[7] BOISTARD Pascal - "Le prix de l'eau", dans "La gestion de l'eau", BARRAQUE Bernard dir, Problèmes Politiques et Sociaux nº86, sept 1992, pp. $14-20$.

[8] COMMISSARIAT GÉNÉRAL DU PLAN - "L'économie face à l'écologie", Rapport de l'atelier présidé par Christian STOFFAËS, La Découverte-La Documentation Française, 1993.

[9] COMMISSARIAT GÉNÉRAL DU PLAN - "Rapport d'Évaluation du dispositif des Agences de l'eau", La Documentation Française, décembre $1997,215 \mathrm{p}$.

[10] COMMISSION MÉDITERRANNÉENNE DU DÉVELOPPEMENT DURABLE - Rapport de synthèse des fiches d'information de l'atelier sur la gestion des demandes en eau, septembre 1997, 10 p.

[11] COUR DES COMPTES - Rapport sur la "gestion des services publics locaux d'eau et d'assainissement “", 1997, 292 p.

[12] EASTER William and HEARNE Robert - "Water markets and decentralized water ressources management : international problems and opportunities", Water Resources Bulletin, volume 31, n 1, February 1995, 12 p.

[13] EASTER W.illiam and HEARNE Robert - "Water Allocation and Water Markets : An Analysis of Gains from Trade in Chile", World Bank Technical Paper n³15, 1995, 75 p.

[14] ERHARD-CASSEGRAIN Annie \& MARGAT Jean - "Introduction l'économie générale de l'eau", Masson, Paris, 1982, 361 p.

[15] EUROSTAF - "La distribution de l'eau en Europe", Eurostaf, collection "Analyses de secteurs", $3^{\text {ème }}$ trimestre 1990.

[16] FAO - Rapport annuel 1993, Politique de l'eau et agriculture.

[17] FAUDRY Daniel - "L'impossible recouvrement des coûts dans les villes latino-américaines", dans "Coût et prix de l'eau en ville : alimentation et assainissement", Actes du colloque organisé par l'École Nationale des Ponts et chaussées, Paris, 6-8 déc 1988, Presses de l'ENPC, 1988, pp. 377. 392.

[18] FREDERICK K. - "Economic instrument for managing water. The US experience". International Conference on "Institutional dimensions of Water Resources Management : Comparative Analysis in the European Union and the United States", Lisboa, Portugal, july 10-12, 1995, 11 p.

[19] GENTY Yves-Noël - "La filière française de l'eau : surmonter les paradoxes", pouvoirs locaux, Les Cahiers de la Décentralisation, $n^{\circ} 8$, mars 1991, pp. 88-91.
[20] HYDROSYSTEMES-INRA - "La valeur économique des hydrosystèmes : apports et diversité de l'approche contingente", Actes du séminaire Hydrosystèmes et Société, Paris 22 décembre 1993, 95 p.

[21] KHOURI Nadim- "Wastewater reuse implementation in selected countries of the Middle-East and North-Africa", Canadian Journal of development Studies , Special Issue, 1992.

[22] LABRE Jacques - "Faut-il craindre une pénurie des ressources en eau au $\mathrm{XX}^{e}$ siècle ? Les thèses en présence in la Houille Blanche $\mathrm{N}^{\circ} 2,1998,2 \mathrm{p}$.

[23] LOCATELLI B., PEIZERAT S., SICARD K., et VILLETTE S. - "Intérêts et obstacles à la mise en cuvre des marchés de droits à polluer". ENGREF, 1994, 75 p.

[24] MEUBLAT Guy - "Sciences économiques, gestion de l'eau, gestion du fleuve" Colloque international "Le fleuve et ses métamorphoses", Lyon. 13 mai 1992,18 p.

[25] MOREL À L'HUISSIER Alain - "Économie de l'eau et société urbaine en Afrique Noire", Pour, $\mathrm{N}^{\circ}$ spécial "Le partage de l'eau", $\mathrm{N}^{\circ}$ 127-128, mars 1991, pp. 49-57.

[26] NICOLAZO Jean-Loïc - "Les Agences de l'eau", $4^{\mathrm{eme} e}$ édition, Paris, P. Johanet et ses fils éditeurs, $207 \mathrm{p}$.

[27] NOWAK Françoise - "Le prix de l'eau", Economica, novembre 1995 $111 \mathrm{p}$.

[28] OCDE - "Tarification des services relatifs à l'eau, Paris, 1987, 165 p.

[29] OCDE - "Gestion de l'eau : performances et défits dans les pays de I'OCDE", 1994

[30] OCDE - Réunion sur la gestion des ressources en eau, 10-11 mai 1994. Conclusions, Comité d'aide au développement-OCDE/TD (94) 106.

[31] OCDE - "Statistiques sur l'aide publique au développement allouée au secteur de l'eau", Conférence internationale "eau et développement durable", Paris 19-20-21 mars 1998

[32] OMS - "World Water, The International Drinking Water Supply alu Sanitation Decade Directory", Londres, 1987.

[33] PARLEMENT EUROPÉEN - "Réseaux hydrauliques", Rapport IZQUIEDO COLLADO, doc. A4-0407/97, le point de la session, janvier 1998.

[34] PENNING-ROWSELL Edmond C - "Trends in water management : french, international and british experiences". Séminaire d'évaluation des politiques d'environnement, Groupe Prospective du Ministère de I'Environnement, 30 jan 1990, $17 \mathrm{p}$.

[35] POQUET Guy - "Perception et attente des consommateurs sur le prix de l'eau", CREDOC, janvier 1997, $39 \mathrm{p}$.

[36] RAINELLI Pierre - "Les bases de l'économie de l'environnement", dans l'eau en Loire-Bretagne : l'économie de l'eau, juillet 1996, $n^{\circ}$ 57, pp 15-17.

[37] RIGGS David - "Market Incentives for Water Quality : A case Study of the Tar - Pamlico River Basin, North Carolina", Center for Policy Studies, Clemson University, South Carolina, december 1993, 40 p.

[38] RINGSKOG Larry and SIMPSON Larry - "Water Markets in the Americas", Directions in Development, The World Bank, 1997, 52 p.

[39] SIMPSON Larry - "Les marchés des droits d'eau aux États-Unis", Revue Française de Géoéconomie n 4, hiver 1997-1998, Economica, 11 p.

[40] VALIRON François - "Les ouvrages nécéssaires pour les réseaux d'alimentation en eau et assainissement : investissement et financement", Colloque "Coût et prix de l'eau de demain", AFPE-AGHTM -Agence de I'Eau Seine-Normandie, Paris, 22 mai 1991, 12 p.

[41] WORLD BANK - "Water Princing Experiences : An International Perspective”. World Bank Technical Paper n ${ }^{\circ} 386,1997,164$ p. 